\title{
Performance of thermal conductivity probes for planetary applications
}

\author{
E. S. Hütter and N. I. Kömle \\ Space Research Institute, Austrian Academy of Sciences, Graz, Austria \\ Correspondence to: N. I. Kömle (norbert.koemle@oeaw.ac.at)
}

Received: 15 December 2011 - Published in Geosci. Instrum. Method. Data Syst. Discuss.: 5 January 2012 Revised: 20 April 2012 - Accepted: 24 April 2012 - Published: 22 May 2012

\begin{abstract}
This work aims to contribute to the development of in situ instruments feasible for space application. Commercial as well as custom-made thermal sensors, based on the transient hot wire technique and suitable for direct measurement of the effective thermal conductivity of granular media, were tested for application under airless conditions. In order to check the ability of custom-made sensors to measure the thermal conductivity of planetary surface layers, detailed numerical simulations predicting the response of the different sensors have been performed. These simulations reveal that for investigations under high vacuum conditions (as they prevail, e.g. on the lunar surface), the derived thermal conductivity values can significantly depend on sensor geometry, axial heat flow, and the thermal contact between probe and surrounding material. Therefore, a careful calibration of each particular sensor is necessary in order to obtain reliable thermal conductivity measurements. The custom-made sensors presented in this work can serve as prototypes for payload to be flown on future planetary lander missions, in particular for airless bodies like the Moon, asteroids and comets, but also for Mars.
\end{abstract}

\section{Introduction}

The physical property which is in the focus of this work is the thermal conductivity of materials composing the solid nearsurface layers of planetary bodies, including the terrestrial planets and their satellites as well as asteroids and comet nuclei. To know this property and its variation with depth is of high interest for a correct understanding of solar system objects and their evolution, for several reasons:
- For modeling the thermal evolution of a planet, the average heat flux across the surface is the decisive boundary condition. In order to determine this property, (i) the average temperature gradient in the near-surface layer (unaffected by diurnal and seasonal variations) and (ii) the thermal conductivity of the near-surface material must be known (Hofmeister et al., 2007).

- Together with other physical material parameters (e.g. mechanical, electrical, optical), thermal properties control to a high extent the processes taking place on a planetary surface. For example, the activity of comets in response to solar irradiation is strongly influenced by the thermal conductivity of the ice/dust mixture at and below the nucleus' surface (Kömle, 2005).

Moreover, variations in thermal conductivity can to some extent be used as an indicator for the presence of water or ice, since dry particulate rock (sand) has a significantly smaller thermal conductivity than water-saturated particulates or ice (Incropera et al., 2007). While for Earth direct (in situ), measurements of thermal conductivity and heat flux are relatively simple, because the material is easy to access and there is little limitation concerning mass and power consumption of the instrumentation, the situation is quite different for other planetary bodies. Although there exist also indirect methods to obtain information on the thermal properties of a planetary surface by measuring the emitted IR-flux from an orbiting satellite, such measurements usually need to be supplemented by local measurements to allow proper calibration. Examples for remote measurements are the data obtained for Mars by the Mars Global Surveyor Thermal Emission Spectrometer (TES) and the Mars Odyssey Thermal Emission Spectrometer (THEMIS) (Mellon et al., 2000). 
In situ measurements of thermal properties on planetary bodies other than Earth are still extremely scarce. To date thermal conductivity (and/or heat flux) data exist only for two terrestrial bodies: Moon and Mars. For the Moon the manned space missions Apollo 15 (1971) and Apollo 17 (1972) carried a heat flow and physical properties package called ALSEP, which was deposited in pre-drilled boreholes of about $2 \mathrm{~m}$ depth on the lunar surface. Both the vertical temperature gradient and the thermal conductivity of the surrounding material was measured (Langseth et al., 1972, 1973).

On Mars the first in situ measurements of thermal properties, covering the top few millimeters of the surface were carried out in 2008 by the Thermal and Electrical Conductivity Probe (TECP) on board the lander Phoenix (Zent et al., 2009). A further in situ instrument for measuring thermal properties is the MUlti-PUrpose Sensor for Surface and SubSurface Science (MUPUS). It is part of the scientific payload on the lander Philae on Rosetta, a cometary mission on its way to the nucleus of comet Churyumov-Gerasimenko, where it is expected to land in late 2014 (Spohn, 2007).

The perhaps most commonly used method to measure the thermal conductivity of soils and sands is the so-called transient line heat source technique (Wechsler, 1992; Kömle et al., 2007). The basic principle of this method is to insert a constant linear heat source into the sample of interest. Simultaneously, the temperature response is measured at or close to the source. The thermal properties of the surrounding material influence the measured temperature increase in a way that under certain conditions the thermal conductivity can be determined from the measured temperature response. Since this method is well approved for thermal conductivity measurements on Earth, it is expedient to use it also on other planetary surfaces. However, to establish successful application of the line heat source method in remote planetary environments demands extensive laboratory testing and modeling work. A comprehensive review of existing prototypes used in or developed for planetary applications was recently given by Kömle et al. (2011).

The present work aims to make a contribution towards the development of a robust and reliable in situ thermal conductivity measurement instrument for application on planetary lander missions. Commercial as well as custom-made sensors were tested. A large number of measurements have been performed on several planetary analogue samples in a vacuum chamber at the Space Research Institute (IWF) Graz under various pressure conditions. While this paper concentrates mainly on modeling aspects, the results of these measurements have been reported in several published papers (Hütter et al., 2008; Kömle et al., 2010) and in a more detailed form in Hütter $(2007,2011)$.

In this paper we study the behavior of the sensors from a theoretical point of view. For this purpose numerical models of the measurement configuration for the particular sensors are set up. Special attention is given to problems which are not that significant in terrestrial environments, but can, as was witnessed during this work, become severe in a high vacuum environment. In particular these are axial heat flow along the sensor caused by the electrical connections, high thermal resistance between the sensor and the surrounding sample material, and radiative interaction between sensor and test specimen.

\section{Theory of transient thermal conductivity probes}

In general, the methods for measuring thermal conductivity can be divided into two groups. The steady state techniques on the one hand and the non-steady state techniques on the other. In case of the steady state methods a constant temperature difference is established in the sample. This method demands high complexity of the measurement system and is therefore unsuitable for application in the field or on space missions. The so-called transient techniques generally use a heater of certain geometry embedded in the sample, which is subsequently heated over a defined time interval. At the same time the temperature is measured at or close to the heater. The temperature response depends on the thermal properties of the surrounding medium. Such measurement systems are less complex than steady state methods and are therefore better qualified for field and space applications. The perhaps most commonly used method for measuring the thermal conductivity of soils and sands in situ is the line heat source technique (Wechsler, 1992). This transient method uses an approximately needle-shaped heater/sensor combination heated by a controlled electrical power. In the following, the theory related to this method is outlined.

The general approach for the line heat source technique is that of an infinite line source embedded in an infinite, homogeneous, and isotropic medium (Carslaw and Jaeger, 1959). For such cylinder-symmetric boundary value problems the heat conduction equation can be written in the form:

$\frac{1}{\mu} \frac{\partial T}{\partial t}=\frac{\partial^{2} T}{\partial r^{2}}+\frac{1}{r} \frac{\partial T}{\partial r}+\frac{Q(r, t)}{k}$

valid over the domain $0<r<\infty$ and $t>0$. Initial and boundary conditions are given as $T=T_{0}$ on the domain $(0<r<\infty, t=0)$ and $T=T_{0}$ for $r \rightarrow \infty, t>0 . Q(r, t)$ denotes a volume heat source $\left[\mathrm{Wm}^{-3}\right], k$ thermal conductivity and $\mu$ thermal diffusivity.

A general solution can be derived by integral transformation (Hankel-transform, see Özisik, 1989):

$$
\begin{gathered}
T(r, t)=\int_{\beta=0}^{\infty}\left[\beta J _ { 0 } ( \beta r ) \operatorname { e x p } ( - \mu \beta ^ { 2 } t ) \left(T_{0} \int_{r^{\prime}=0}^{\infty} r^{\prime} J_{0}\left(\beta r^{\prime}\right) \mathrm{d} r^{\prime}\right.\right. \\
\left.\left.+\frac{\mu}{k} \int_{r^{\prime}=0}^{\infty} r^{\prime} J_{0}\left(\beta r^{\prime}\right) Q\left(r^{\prime}, t^{\prime}\right) \mathrm{d} r^{\prime}\right)\right] \mathrm{d} \beta
\end{gathered}
$$


where $J_{0}(x)$ is the Bessel function of the first kind and zero'th order.

\subsection{Continuous line heat source}

For the case of a continuous line heat source $Q_{1}\left[\mathrm{Wm}^{-1}\right]$ in the center $(r=0)$ that supplies energy at a constant rate, the volume power density $Q(r, t)\left[\mathrm{Wm}^{-3}\right]$ can be specified in the form:

$Q(r, t)=\frac{Q_{1}}{2 \pi r} \delta(r-0)$

where $\delta(r-0)$ is the Dirac delta function at the position $r=0$. Substituting Relation (3) into Eq. (2) leads to the expression for the temperature response due to heat emitted from the line source:

$$
\begin{aligned}
& T(r, t)=T_{0}+\frac{Q_{1}}{4 \pi k} \int_{t^{\prime}=0}^{t} \frac{1}{\left(t-t^{\prime}\right)} \exp \left(-\frac{r^{2}}{4 \mu\left(t-t^{\prime}\right)}\right) \mathrm{d} t^{\prime} \\
& \quad=T_{0}+\frac{Q_{1}}{4 \pi k} \operatorname{Ei}\left(-\frac{r^{2}}{4 \mu t}\right)
\end{aligned}
$$

The expression $-\operatorname{Ei}(-x)=\int_{x}^{\infty} \frac{\exp (-u)}{u} \mathrm{~d} u$ in Eq. (4) denotes the Exponential Integral Function. This integral can be expressed by a series expansion (Abramowitz and Stegun, 1964) as

$$
\begin{aligned}
& -\operatorname{Ei}(-x)=-\zeta-\ln x-\sum_{n=1}^{\infty} \frac{(-1)^{n} x^{n}}{n n !}=-\zeta-\ln x \\
& +x-\frac{1}{4} x^{2}+\ldots
\end{aligned}
$$

where $\zeta=0.5772$ denotes the Euler constant. For small $x$ values (equivalent to large values of $t$ ) the contributions after the logarithmic term can be neglected and the temperature change can be approximated by

$T(r, t)=\frac{Q_{1}}{4 \pi k}\left(-\zeta+\ln t+\ln \frac{4 \mu}{r^{2}}\right)$.

Thus the variation of temperature with the natural logarithm of time as the independent variable is

$\frac{\mathrm{d} T}{\mathrm{~d} \ln t}=\frac{Q_{1}}{4 \pi k}$

In Eq. (7) the temperature rise due to heating depends only on the applied amount of heat $Q_{1}$ (heating power per unit length of the line source positioned at $r=0$ ), the thermal conductivity $k$ of the surrounding medium, and the time interval used. This approximation gives a linear relation between the temperature change along the line heat source and the natural logarithm of time. From that the thermal conductivity can be derived if heating power and temperature increase as a function of time are known.

\subsection{Continuous cylindrical surface source}

A second problem of interest is that of a continuous cylindrical surface heat source $Q_{\text {cyl }}\left[\mathrm{Wm}^{-1}\right]$ with a radius $a$. (This satisfies the geometry of a thermal conductivity probe better than the line heat source approach). Following a similar approach as for the line heat source, $Q(r, t)$ is specified as

$Q(r, t)=\frac{Q_{\mathrm{cyl}}}{2 \pi r} \delta(r-a)$.

The solution for the temperature distribution due to a constant continuous cylindrical surface source at $r=a$ can now again be obtained from Eq. (2) by integration (Özisik, 1989):

$$
\begin{aligned}
& T(r, t)=\frac{Q_{\mathrm{cyl}}}{4 \pi k} \int_{t^{\prime}=0}^{t} \frac{1}{\left(t-t^{\prime}\right)} \exp \left(-\frac{r^{2}+a^{2}}{4 \mu\left(t-t^{\prime}\right)}\right) \\
& \quad I_{0}\left(\frac{r a}{2 \mu\left(t-t^{\prime}\right)}\right) \mathrm{d} t^{\prime}
\end{aligned}
$$

where $I_{0}(x)$ is the modified Bessel function of zero'th order, which can also be expressed by a series expansion of the form:

$$
\begin{aligned}
& I_{0}(x)=\sum_{n=0}^{\infty} \frac{1}{(n !)^{2}}\left(\frac{x^{2}}{2}\right)=1+\left(\frac{x}{2}\right)^{2}+\frac{1}{4}\left(\frac{x}{2}\right)^{4} \\
& +\frac{1}{36}\left(\frac{x}{2}\right)^{6}+\ldots
\end{aligned}
$$

Using the identity

$$
I_{0}(x)=1+\left[I_{0}(x)-1\right]
$$

Equation (9) may be split up into two contributions:

$$
\begin{aligned}
T(r, t)= & \frac{Q_{\mathrm{cyl}}}{4 \pi k} \int_{t^{\prime}=0}^{t} \frac{1}{\left(t-t^{\prime}\right)} \exp \left(-\frac{r^{2}+a^{2}}{4 \mu\left(t-t^{\prime}\right)}\right) \mathrm{d} t^{\prime} \\
+ & \frac{Q_{\mathrm{cyl}}}{4 \pi k} \int_{t^{\prime}=0}^{t} \frac{1}{\left(t-t^{\prime}\right)} \exp \left(-\frac{r^{2}+a^{2}}{4 \mu\left(t-t^{\prime}\right)}\right) \\
& {\left[I_{0}\left(\frac{r a}{2 \mu\left(t-t^{\prime}\right)}\right)-1\right] \mathrm{d} t^{\prime} . }
\end{aligned}
$$

The first term of the integral solution given in Eq. (12) is the solution for a line heat source displaced by about the radius of the cylindrical surface source. The second term contains the influence stemming from the cylindrical nature of the source. The temperature increase due to heating at the source $r=a$ is obtained as 


$$
\begin{aligned}
T(t)= & \frac{Q_{\text {cyl }}}{4 \pi k} \int_{t^{\prime}=0}^{t} \frac{1}{\left(t-t^{\prime}\right)} \exp \left(-\frac{a^{2}}{2 \mu\left(t-t^{\prime}\right)}\right) \mathrm{d} t^{\prime} \\
+ & \frac{Q_{\text {cyl }}}{4 \pi k} \int_{t^{\prime}=0}^{t} \frac{1}{\left(t-t^{\prime}\right)} \exp \left(-\frac{a^{2}}{2 \mu\left(t-t^{\prime}\right)}\right) \\
& {\left[I_{0}\left(\frac{a^{2}}{2 \mu\left(t-t^{\prime}\right)}\right)-1\right] \mathrm{d} t^{\prime} . }
\end{aligned}
$$

Equation (13) is of interest for thermal conductivity measurements with hollow cylindrical sensors where the temperature response to heating is measured in the close vicinity of the heater. This applies for the LNP01 probe introduced in Sect. 3 .

In Fig. 1 the integral solution for a continuous cylindrical surface source is shown along with the two parts the integral can be divided into (see Eq. 13). The parameters used for this graph are those for a measurement with the hollow cylindrical sensor LNP01 in Agar. It can be seen that at small times the solution is dominated by the cylindrical part of Eq. (13) while for times of about $1000 \mathrm{~s}$ and larger the term corresponding to a line heat source controls the temperature distribution.

\subsection{Consideration of heat source properties}

The theory introduced in the previous paragraphs examines infinite samples and infinitely long and thin sensors with therefore negligible thermal properties. The contact between sensor and medium is considered to be ideal. However, this is not the case for real measurement devices. Solutions of the heat equation for the more realistic setup of a finite probe and non-ideal contact between sensor and probe have been developed and discussed amongst others by Jaeger (1956), Blackwell (1954), and de Vries and Peck (1958). The most comprehensive presentation on the theory of cylinder-symmetric heat sources can be found in the classical textbook of Carslaw and Jaeger (1959). In the following the relations given in Jaeger (1956) are outlined. A general integral solution was derived for the problem of a perfectly conducting cylinder of a certain radius $r_{\mathrm{sen}}$, heat capacity $c_{\mathrm{sen}}$ and density $\varrho_{\text {sen }}$, embedded in an infinite medium with known properties. The intersection between sensor and medium is controlled by a contact resistance per unit area given as $1 / H$, where $H$ is defined as the contact conductance. The general solution given in Eq. (18) is expressed in terms of the dimensionless parameters $\tau, \alpha$ and $h$, which are defined as

$$
\begin{aligned}
& \tau=\frac{\mu t}{r_{\mathrm{sen}}^{2}} \\
& \alpha=\frac{2 \pi r_{\mathrm{sen}}^{2} \varrho c}{S} \\
& h=\frac{k}{r_{\mathrm{sen}} H}
\end{aligned}
$$

Thermal diffusivity $\mu$, density $\varrho$, specific heat $c$ and thermal conductivity $k$ are the medium properties. $S$ is the heat capacity of the probe per unit length and can be expressed as

$S=A_{\operatorname{sen}} c_{\text {sen }} \varrho_{\text {sen }}$

with $A_{\text {sen }}$ denoting the cross-section area of the sensor.

A dimensionless integral solution can be derived as (Jaeger, 1956)

$$
\frac{T k}{Q_{1}}=G(h, \alpha, \tau)=\frac{2 \alpha^{2}}{\pi^{3}} \int_{0}^{\infty} \frac{\left[1-\exp \left(-\tau x^{2}\right)\right] \mathrm{d} x}{x^{3} \Delta(x)}
$$

with

$$
\begin{gathered}
\Delta(x)=\left[x J_{0}(x)-\left(\alpha-h x^{2}\right) J_{1}(x)\right]^{2}+ \\
{\left[x Y_{0}(x)-\left(\alpha-h x^{2}\right) Y_{1}(x)\right]^{2}}
\end{gathered}
$$

$J_{0}$ and $J_{1}$ are the Bessel functions of the first kind of order 0 and 1 , while $Y_{0}$ and $Y_{1}$ are Bessel functions of the second kind of order 0 and 1 . Approximations for the function $G(h, \alpha, \tau)$ can be obtained by series expansions of the integral solution (18). Of particular interest is the long time approximation:

$G(h, \alpha, \tau)=\frac{1}{4 \pi}\left[2 h+\ln \frac{4 \tau}{C}-\frac{(4 h-\alpha)}{2 \alpha \tau}+\frac{(\alpha-2)}{2 \alpha \tau} \ln \frac{4 \tau}{C}\right]$

where $C=\exp (\zeta)=1.7811$ with the Euler constant $\zeta=$ 0.5772 . The derivation of Relation (20) from the general solution (18) is given in more detail in the classical papers by Blackwell (1954) and in Jaeger (1956). For the limiting case of a very thin sensor and vanishing contact resistance Relation (20) becomes Relation (6). The given equations can be used to simulate the temperature response due to heating for known properties of sample and sensor. Although the short time and long time evaluation formulas are still useful for doing estimates, it should also be noted that they have been derived at a time when the numerical evaluation of integrals containing Bessel functions was still extremely cumbersome and time consuming. With today's computing possibilities the best way to an accurate evaluation is to solve the integral in Eq. (18) numerically and to use built-in subroutines to compute the Bessel functions under the integral. In our work we used mainly the MATLAB programming language to perform these tasks. 


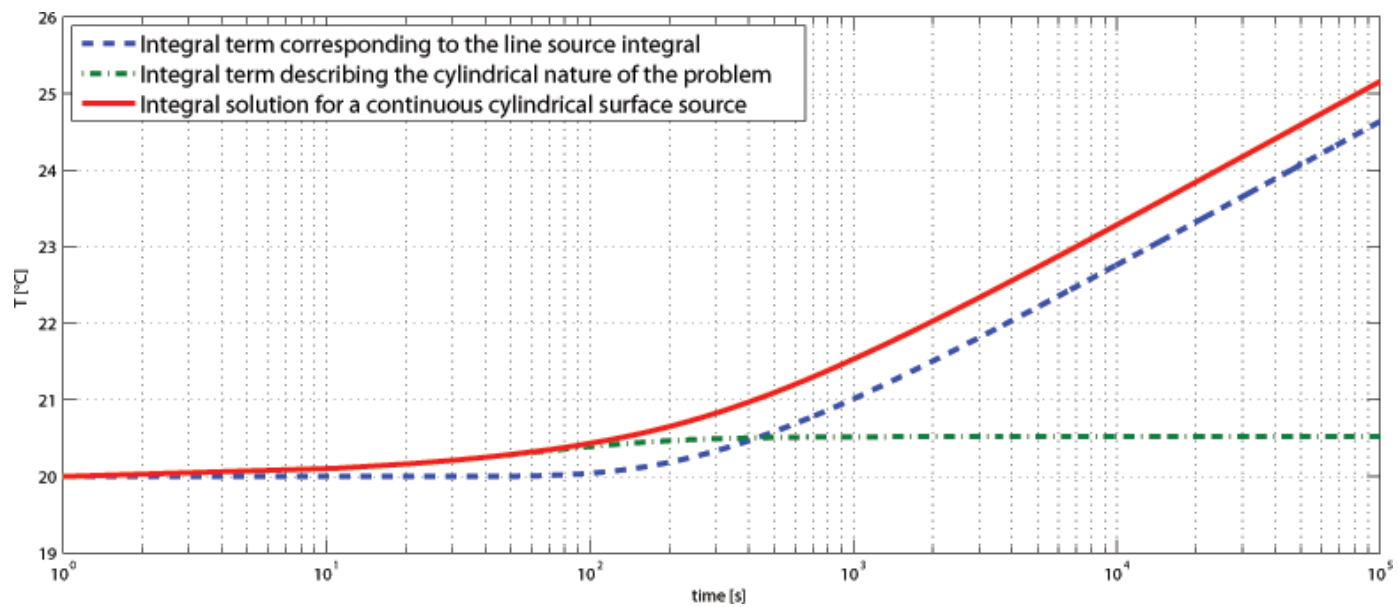

Fig. 1. Integral solutions for a continuous cylindrical surface source. The used parameters match those of a measurement with LNP01 performed in Agar. $\left(r_{1}=0.0075 \mathrm{~mm} ; Q_{\text {cyl }}=6.15 \mathrm{Wm}^{-1} ; k=0.6 \mathrm{Wm}^{-1} \mathrm{~K}^{-1} ; \mu=11 \times 10^{-8} \mathrm{~m}^{2} \mathrm{~s}^{-1}\right)$.

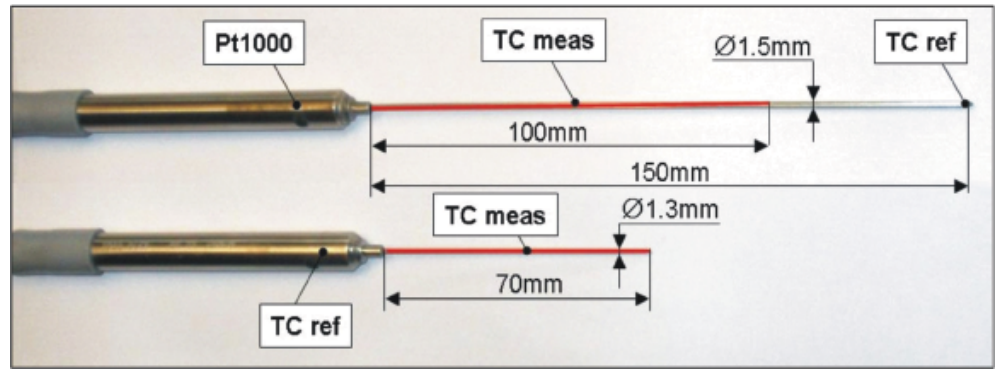

Fig. 2. The commercial TP02 (long needle) and TP08 (short needle) thermal conductivity probes produced by the Dutch company Hukseflux. The heated part is indicated in red.

\section{Measurement probes}

The geometry and principle of the transient hot wire technique as outlined in the previous sections allows bottom-ofthe-line field application. This method has been assigned for a broad application range. The commercial needle sensors TP02 and TP08 investigated in this work are suitable for various granular materials like sands, soils and powders. Such a needle-shaped sensor could be placed at the tip of an robotic arm, as the thermal and electrical conductivity probe TECP on the Mars polar lander Phoenix. However, for this application a sensor of several centimeter length should be robust and resistant against bending. The TP02 and TP08 do not fulfil this requirement. During investigation of the particulate rock material with grain sizes larger than one millimeter it was noted that the commercial sensors easily got stuck and tended to be deformed by bending. The more robust prototypes LNP02 and LNP03 were not affected by this problem and are therefore more suitable for granular rock.

The third kind of sensor investigated is the LNP01, which is a large hollow cylindrical sensor. Such a sensor could be implemented into a drilling rod. Further, this sensor concept is similar to the thermal property measurement sys- tem implemented in the hull of the $\mathrm{HP}^{3}$-instrument, a moletype probe for subsurface exploration ${ }^{1}$. All sensors used and tested in this work were built by the Dutch company Hukseflux (www.hukseflux.com).

\subsection{Commercial line heat source probes (TP02, TP08)}

The TP02 and TP08, both shown in Fig. 2, are commercial needle probes for thermal conductivity determination based on the transient hot wire theory. The TP02 has a length of $150 \mathrm{~mm}$ and a diameter of $1.5 \mathrm{~mm}$. It is heated over two thirds of its length by an embedded constantan heating wire. The TP08 needle has a length of $70 \mathrm{~mm}$ and a diameter of $1.3 \mathrm{~mm}$. In contrast to the TP02 it is heated over the whole length by a constantan heating wire. Both sensors measure the temperature response to heating by means of thermocouples. The TP02 has one thermocouple junction positioned $50 \mathrm{~mm}$ from the base and a reference junction placed in the unheated tip. Additionally, a temperature dependent resistor (Pt1000) is embedded in the base.

\footnotetext{
${ }^{1}$ http://www.dlr.de/dlr/en/desktopdefault.aspx/tabid-10255/ 365_read-818
} 


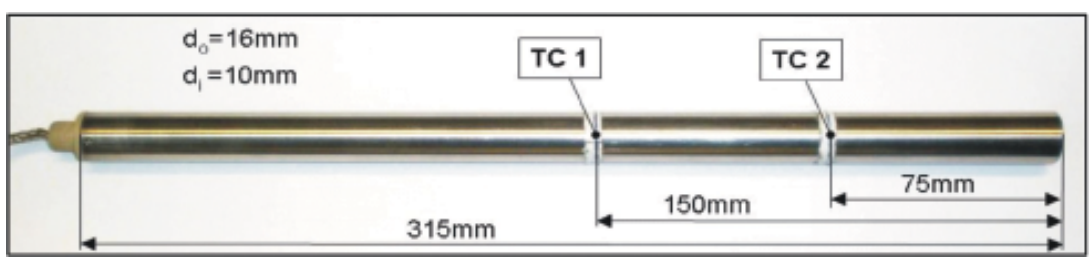

Fig. 3. The thermal conductivity probe prototype LNP01. This hollow cylindrical sensor is heated over its whole length.

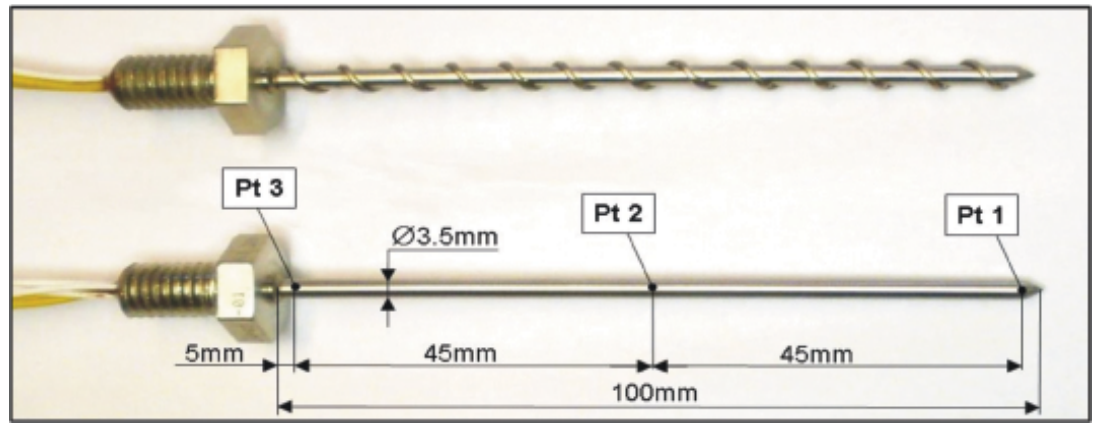

Fig. 4. The LNP02 (needle with winding) and LNP03 (plane needle) prototype.

The TP08 has a thermocouple junction embedded $32 \mathrm{~mm}$ from the tip of the sensor and the reference junction is placed in the base (thicker upper part). Therefore, it is reasonable to place the TP08 deep enough inside the sample, so that the base is also covered with material and can not be disturbed by the ambient temperature fluctuations. Both sensors use thermocouples of type $\mathrm{K}$.

\subsection{Hollow cylindrical prototype (LNP01)}

This relatively large sensor is very robust and is suitable for the investigation of coarse-grained material in the millimeter to centimeter size range. The LNP01 sensor, shown in Fig. 3, is shaped as a hollow cylinder with a length of $315 \mathrm{~mm}$, an outer diameter of $16 \mathrm{~mm}$ and an inner diameter of $10 \mathrm{~mm}$. Heating is done by means of a Kapton heating foil embedded between the two concentric steel tubes of the hollow cylinder, with an electrical resistance of about $154 \Omega \mathrm{m}^{-1}$. The heating foil covers the whole circumference of the sensor. The probe is closed at one end by soldering. The free space between the concentric tubes is filled up with epoxy resin, while the interior of the sensor remains hollow (optionally it can be filled up by sample material). For differential temperature measurement thermocouple junctions are located in the middle and near the bottom end of the LNP01, respectively. The power for heating is supplied by an external constant current source which allows free adjustment of the heating power.

\subsection{Short and robust prototypes (LNP02, LNP03)}

The LNP02 and LNP03 sensors, shown in Fig. 4, are of comparable size as the commercial TP08 thermal conductivity probe, but more robust and can hardly be bent when pushed into granular or slightly cohesive material. LNP02 has the shape of a needle with a screw-like winding, its mean diameter is $4.5 \mathrm{~mm}$, while LNP03 is a needle with a circular cross section over its whole length with a diameter of $3.5 \mathrm{~mm}$. Both sensors have a length of $100 \mathrm{~mm}$ and are heated over the whole length by a constantan heating wire. For each probe the temperature response to heating is measured by three temperature dependent resistors (Pt1000). These are placed at the tip, the middle and the base of the sensor. The temperature measurement is done in a four wire configuration.

\subsection{Constraints}

Naturally, the thermal conductivity probes used for real measurements deviate from the theoretical assumption for a transient line heat source as outlined in Sect. 2. They have finite dimensions and non-negligible properties. The investigated samples are, especially in the case of laboratory measurements, of limited size and the contact between measurement probe and specimen is also not ideal. These limitations lead to some constraints that have to be considered.

\subsubsection{Transient time after onset of heating}

The time period $t_{\text {trans }}$ is an estimate for the duration of the nonlinear part of the temperature versus $\ln t$ curve after the onset of heating. This nonlinear part is mainly due to the initial heating of the finite sensor volume, since the heat wave 

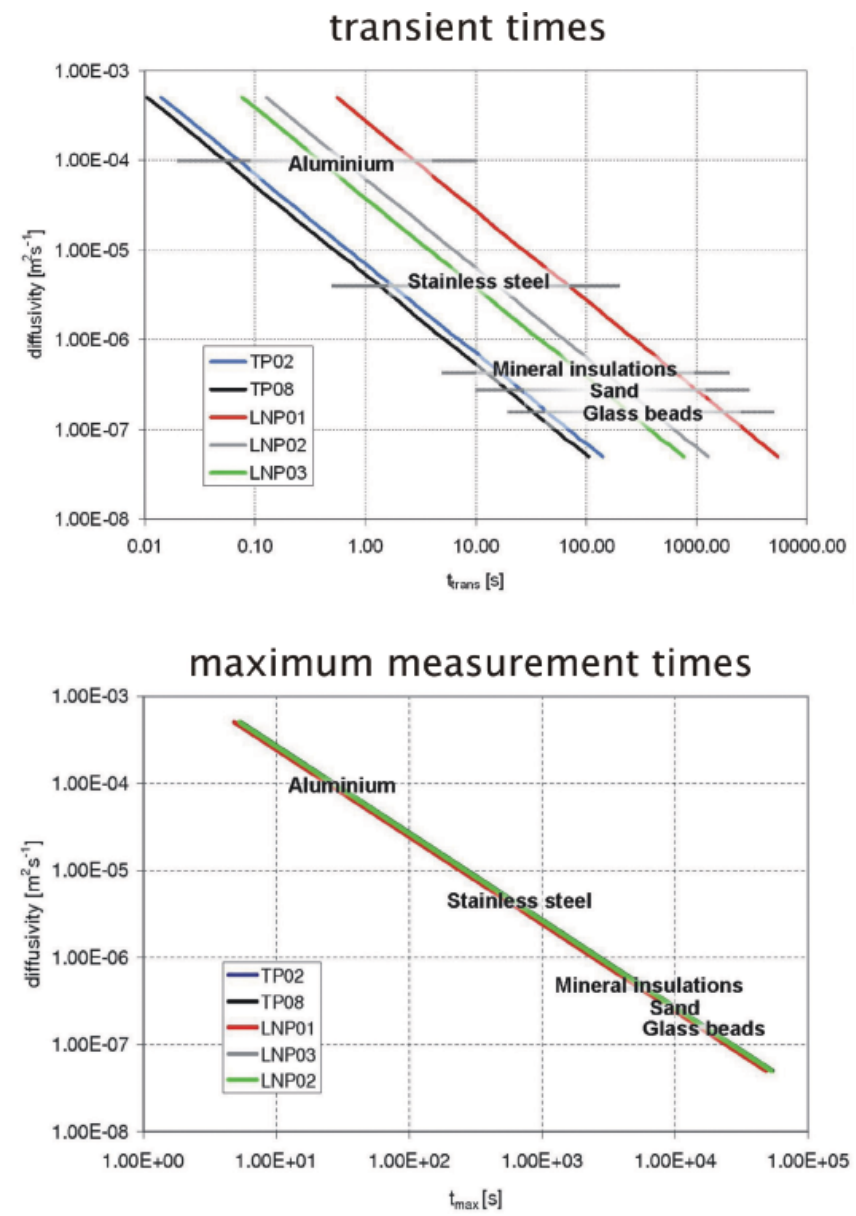

Fig. 5. Transient times and maximum measurement times calculated for the five investigated thermal probes using a diffusivity range from $\mu=8 \times 10^{-4} \mathrm{~m}^{2} \mathrm{~s}^{-1}$ to $\mu=5 \times 10^{-8} \mathrm{~m}^{2} \mathrm{~s}^{-1}$.

has to propagate through the sensor material first. It also depends on the thermal properties of the surrounding medium, because for a proper measurement, an adequate specimen volume has to be sampled. Additionally, the transient time is influenced by the contact resistance. It was shown by Vos (1955) and Goodhew and Griffiths (2004) that the minimum nonlinear time period can be estimated as

$t_{\text {trans }} \geq \frac{50 R_{\text {sen }}^{2}}{4 \mu_{\text {sample }}}$.

Relation (21) is valid for uniform heating of a thin cylindrical sensor like, e.g., the TP02. However, for the LNP01 sensor, that has the shape of a hollow cylinder, an effective sensor radius has to be defined (Kömle et al., 2010). This is done by deriving an equivalent cylinder radius for the volume of the hollow cylinder. The volume of the hollow cylinder $V_{\mathrm{hc}}$ is given by

$V_{\mathrm{hc}}=\left(R_{\mathrm{O}}^{2}-R_{\mathrm{I}}^{2}\right) \pi l$ where $l$ is the length of the sensor and $R_{\mathrm{I}}$ and $R_{\mathrm{O}}$ are the inner and outer radius. The volume of the equivalent full cylinder $V_{\mathrm{c}}$ is

$V_{\mathrm{c}}=R_{\mathrm{eq}}^{2} \pi l$

with $R_{\text {eq }}$ being the equivalent radius. Equating Eqs. (22) and (23) leads to the following expression for the equivalent radius:

$R_{\mathrm{eq}}=\sqrt{\left(R_{\mathrm{O}}^{2}-R_{\mathrm{I}}^{2}\right)}$.

For the LNP01 sensor one obtains $R_{\mathrm{eq}}=4.7 \mathrm{~mm}$. The transient times as calculated by Eq. (21) for the different sensors and a selection of different materials are plotted in Fig. 5 (top panel). As expected, the longest transient times were derived for the largest sensor and the shortest transient times were found for the smallest sensor.

\subsubsection{Maximum measurement time}

The finite dimensions of the investigated samples result in a limitation of the measurement time. The reason is that the heat wave induced by the measurement reaches the sample boundaries sooner or later, depending on the sample thermal properties. From this moment on the temperature distribution in the sample is disturbed and in succession also the measurement. The point in time when the heat wave reaches the sample boundary depends on the distance of the sensor from the boundary and on the thermal diffusivity of the specimen. This maximum measurement time can be estimated by using the following relation (Vos, 1955; Goodhew and Griffiths, 2004):

$t_{\max }=\frac{0.6\left(r_{\text {sample }}-r_{\text {sen }}\right)^{2}}{4 \mu_{\text {sample }}}$.

In Fig. 5 (bottom panel) the maximum measurement times derived for the sensors used in this work are plotted. The presented thermal diffusivity range of $\mu=8 \times 10^{-4} \mathrm{~m}^{2} \mathrm{~s}^{-1}$ to $\mu=5 \times 10^{-8} \mathrm{~m}^{2} \mathrm{~s}^{-1}$ is representative for good and bad thermal conductors. Selected materials are indicated in the plots. In Table 1 the time limits determined for the calibration materials Agar, Teflon, PMMA and Glycerin are given. Not all sensors were tested on all materials due to limitations in sample size. Nevertheless, the transient and maximum measurement times were calculated. The problems that would be caused by inadequate sample size are reflected, e.g. by the determined time limits for the LNP01 in PMMA and Glycerin. In these cases the transient time is larger than the maximum measurement time, so it would never be possible to reach the linear temperature versus $\ln t$ region in a measurement before it would be disturbed by boundary effects. 
Table 1. Maximum and minimum measurement times estimated for the different sensors when used in various calibration materials.

\begin{tabular}{|c|c|c|c|c|c|}
\hline Sensor & Sample & $\begin{array}{c}r_{\text {sample }} \\
{[\mathrm{m}]}\end{array}$ & $\begin{array}{l}\mu_{\text {sample }} \\
{\left[\mathrm{m}^{2} \mathrm{~s}^{-1}\right]}\end{array}$ & $\begin{array}{c}t_{\text {trans }} \\
{[\mathrm{s}]}\end{array}$ & $\begin{array}{r}t_{\max } \\
{[\mathrm{s}]}\end{array}$ \\
\hline \multirow{4}{*}{$\begin{array}{c}\text { TP02 } \\
d_{\text {sen }}=1.5 \times 10^{-3} \mathrm{~m}\end{array}$} & Agar & 0.135 & $14 \times 10^{-8}$ & 50 & 19000 \\
\hline & Teflon & 0.050 & $12 \times 10^{-8}$ & 59 & 3030 \\
\hline & PMMA & 0.030 & $10 \times 10^{-8}$ & 70 & 1350 \\
\hline & Glycerin & 0.040 & $09 \times 09^{-8}$ & 78 & 2670 \\
\hline \multirow{4}{*}{$\begin{array}{c}\text { TP08 } \\
d_{\text {sen }}=1.3 \times 10^{-3} \mathrm{~m}\end{array}$} & Agar & 0.135 & $14 \times 10^{-8}$ & 38 & 19340 \\
\hline & Teflon & 0.050 & $12 \times 10^{-8}$ & 44 & 3040 \\
\hline & PMMA & 0.030 & $10 \times 10^{-8}$ & 53 & 1290 \\
\hline & Glycerin & 0.040 & $09 \times 09^{-8}$ & 59 & 2580 \\
\hline \multirow{4}{*}{$\begin{array}{c}\text { LNP01 } \\
d_{\text {sen }}=9.4 \times 10^{-3} \mathrm{~m}\end{array}$} & Agar & 0.135 & $14 \times 10^{-8}$ & 1972 & 18200 \\
\hline & Teflon & 0.050 & $12 \times 10^{-8}$ & 2300 & 2570 \\
\hline & PMMA & 0.030 & $10 \times 10^{-8}$ & 2760 & 3080 \\
\hline & Glycerin & 0.040 & $09 \times 09^{-8}$ & 3068 & 1070 \\
\hline \multirow{4}{*}{$\begin{array}{c}\text { LNP02 } \\
d_{\text {sen }}=4.5 \times 10^{-3} \mathrm{~m}\end{array}$} & Agar & 0.135 & $14 \times 10^{-8}$ & 452 & 18900 \\
\hline & Teflon & 0.050 & $12 \times 10^{-8}$ & 527 & 2850 \\
\hline & PMMA & 0.030 & $10 \times 10^{-8}$ & 633 & 1160 \\
\hline & Glycerin & 0.040 & $09 \times 09^{-8}$ & 703 & 2380 \\
\hline \multirow{4}{*}{$\begin{array}{c}\text { LNP03 } \\
d_{\text {sen }}=3.5 \times 10^{-3} \mathrm{~m}\end{array}$} & Agar & 0.135 & $14 \times 10^{-8}$ & 273 & 19000 \\
\hline & Teflon & 0.050 & $12 \times 10^{-8}$ & 319 & 2910 \\
\hline & PMMA & 0.030 & $10 \times 10^{-8}$ & 382 & 1200 \\
\hline & Glycerin & 0.040 & $09 \times 09^{-8}$ & 425 & 2440 \\
\hline
\end{tabular}

\subsection{Data evaluation}

Figure 6 shows the characteristics of a measured temperature response to heating versus the natural logarithm of time after Jones (1988). Three parts can be identified in this figure.

First, an initial phase after the onset of heating, which is dominated by the self-heating of the sensor and contact resistance, is observed. This is followed by a section where the temperature versus natural logarithm of time data are linear. This is the part needed for the determination of the thermal conductivity, if the long time approximation given in Eq. (6) is used. From the slope of the temperature versus $\ln t$ data, thermal conductivity can be calculated by using Eq. (7). In the adjacent third part the data are nonlinear again. In this domain, effects of finite sample dimensions and axial errors become important and the data can no longer be evaluated by using Eq. (7). An increase of the measurement curve in the third part as shown in Fig. 6 can, for example, be observed if the wall of the sample container is reached by the heat wave and the conductivity of the container material is lower than that of the sample. A decrease in this part is observed if convection starts to play a role or if the conductivity of the container wall is higher than the sample conductivity.

During a measurement the temperature versus time series with and without heating is collected by a data logger suitable for the particular sensor. From the logged temperaturetime data, the heating current, and the heater resistance, the thermal conductivity is determined. The information about the samples allows to estimate transient and maximum measurement times, which subsequently can be used to narrow down the part of the measurement curve used for evaluation. Finally, the data can be evaluated in three ways that are in the following outlined in more detail.

\subsubsection{Linear regression}

From the linear part of the temperature versus natural logarithm of time data the thermal conductivity is calculated using a linear fit of the form

$T(t)=A+B \ln t$

where the coefficient $B$ corresponds to the right side of Eq. (7), and therefore, the thermal conductivity can be derived as

$k=\frac{P}{4 \pi B}$

where $P$ is the constant heating power per unit length applied to the sensor during the measurement. In general, the linear part to be used for the evaluation lies in between the nonlinear sections, as sketched in Fig. 6. Thus, the challenge of the data interpretation lies in identifying the linear part of the curve. For this purpose the transient time $t_{\text {trans }}$, giving the 


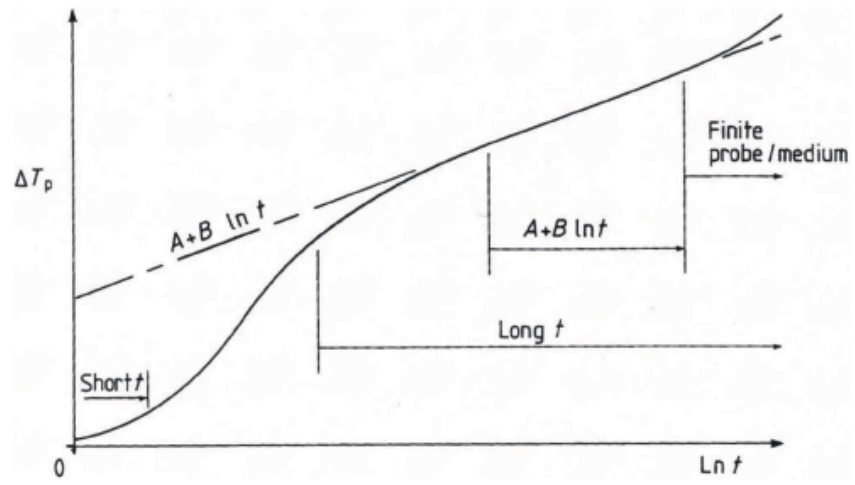

Fig. 6. Qualitative heating curve measured with a line heat sensor (after Jones, 1988).

minimum duration of the nonlinear part after onset of heating, and the maximum measurement time $t_{\max }$, giving the time after which the heat wave reaches the sample boundary, are used as criteria to constrain the evaluation interval. On the time interval defined by these two boundaries a linear least squares fit is performed.

\subsubsection{Successive linear regression}

Since $t_{\text {trans }}$ and $t_{\max }$ can only be estimated, they might not always apply to the measured data. The uncertainties for transient and maximum time lie in the estimates available for the sample diffusivity and the usually unknown magnitude of the contact resistance. Furthermore, axial losses or the onset of convection could reduce the duration of the linear part. To minimize errors in the determined thermal conductivity arising from these effects, successive linear regression can be used.

For this purpose the part of the measurement curve starting from $t_{\text {trans }}$ and the corresponding temperature is divided into sub-intervals. On these curve-sections linear least squares fits are performed. For the measurements evaluated a fit interval of 10 measurement points (this corresponds generally to a time interval of $10 \mathrm{~s}$ in our data sets) were chosen. It was assumed that the majority of the successively determined slopes/conductivities can be derived from the linear part of the measurement curve. Thus, the median of these values should give a good estimate for the conductivity.

\subsubsection{Nonlinear regression}

A nonlinear long time approximation for the heating curve, considering a probe with finite diameter and non-negligible properties and contact resistance between sample and probe, has been used for evaluation, for example, by Jones (1988), Koski and McVey (1983) and Erbas (2001). This relation is a better approximation of the measurement situation and therefore should yield better values. The regression equation has the form

$T(t)=A+B \ln t+C \frac{\ln t}{t}+D \frac{1}{t}$

where terms of the order $\left(1 / t^{2}\right)$ and higher are neglected. The coefficients in Eq. (28) can be expressed as follows:

$$
\begin{aligned}
A= & \frac{P}{4 \pi k}\left[\ln \left(\frac{4 \mu}{r_{\mathrm{sen}}^{2}}\right)-\zeta+\frac{2 k}{r_{\mathrm{sen}} H}\right] \\
B= & \frac{P}{4 \pi k} \\
C= & \frac{P}{4 \pi k} \frac{r_{\mathrm{sen}}^{2}}{2 \mu}\left(1-\frac{\mu S}{\pi r_{\mathrm{sen}}^{2} k}\right) \\
D= & \frac{P}{4 \pi k} \frac{r_{\mathrm{sen}}^{2}}{2 \mu}\left\{\ln \left(\frac{4 \mu}{r_{\mathrm{sen}}^{2}}\right)+(1-\zeta)-\frac{\mu S}{\pi r_{\mathrm{sen}}^{2} k}\right. \\
& {\left.\left[\ln \left(\frac{4 \mu}{r_{\mathrm{sen}}^{2}}\right)-\zeta+\frac{2 k}{r_{\mathrm{sen}} H}\right]\right\} . }
\end{aligned}
$$

The regression Eq. (28) is equivalent to Eq. (20). The nonlinear fit is usually applied on a pre-defined interval of the measurement data beyond the transient region, similar as it is done for the linear fit. For the nonlinear fit procedure a built-in MATLAB function can be used. While from the linear regression no information on the contact resistance $(1 / H)$ can be derived, the nonlinear formula allows to obtain also information on this often poorly constrained parameter, in addition to evaluating the thermal conductivity of the surrounding material.

\section{Numerical modeling of the sensor response}

In order to better understand the temperature response of the sensors, the three types of line heat sensors investigated in this work were modeled with the finite element modeling and simulation software Comsol Multiphysics ${ }^{2}$. In this way the behavior of the sensors could be studied in more detail, and it was possible to access measurement scenarios that could not be experimentally realized with the equipment at hand (for example measurement times of several hours to days). Special attention was given to the factors influencing the measurements under low pressure. The features implemented were (i) the possibility of axial heat flow via the electrical wires leaving the sensor, (ii) the contact resistance at the sensor surface-sample interface and (iii) the possibility of radiation interaction in a layer between sensor and sample, which stands for the clearances between sensor surface and particles that unavoidably develop in a granular material.

To study the influence of these factors the temperature curves resulting from particular settings were computed for the positions of the temperature sensors in the real instruments. The resulting data were processed in such a way that it could be evaluated with the same MATLAB-routine as used

\footnotetext{
${ }^{2}$ www.comsol.com
} 


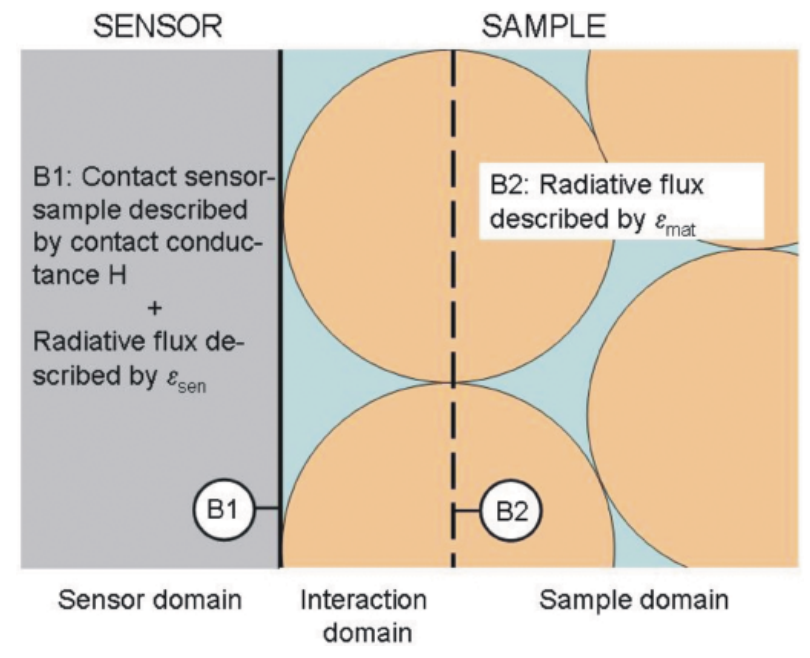

Fig. 7. Schematic of the heat transfer mechanisms between sensor and sample.

for the evaluation of the measurement data obtained from the sensors. Models were set up for the TP02, LNP01 and LNP03 sensors. The results obtained are outlined in more detail in the following paragraphs. For the given geometries and parameters the time-dependent heat conduction equation for a solid body was solved. The general form of the differential equation solved is

$\varrho c \frac{\partial T}{\partial t}+\nabla(-k \nabla T)=Q$.

The problem of the transient line heat source has radial symmetry. Thus, the models were built in the axi-symmetric mode. The following features were implemented for each sensor:

Heating: in the domain representing the heater, a constant amount of energy is continuously released over the measurement time (realized by the volumetric source term $\left.Q\left[\mathrm{Wm}^{-3}\right]\right)$. To get the heating power in $\mathrm{Wm}^{-1}$, as it is recorded in the respective real measurements, the volumetric source term $Q$ has to be multiplied by the cross section of the heater.

Axial heat flow: the unavoidable fact of axial heat flow occurring in a probe due to its construction is considered by modeling the base of the particular probe and the electrical wires that connect the sensors with the electronics and power supply. The wires are represented by a domain of certain height attached to the heater domain, with a continuity condition at the heater-wire boundary and a constant temperature condition at the opposite boundary. As constant temperature the initial temperature $T_{\text {init }}$ was used.

Contact resistance: the contact resistance is modeled using a Neumann boundary condition at the intersection of sensor surface and material:

$-\boldsymbol{n}\left(\boldsymbol{q}_{1}-\boldsymbol{q}_{\mathbf{2}}\right)=H\left(T_{\infty}-T\right)$.
Table 2. Measurement scenarios addressed for each of the three sensor geometries and sample conductivities.

\begin{tabular}{cccc}
\hline No & contact & cable & radiation \\
\hline 1 & poor & no & no \\
2 & good & no & no \\
3 & poor & yes & no \\
4 & good & yes & no \\
5 & no & no & yes \\
6 & no & yes & yes \\
7 & poor & yes & yes \\
\hline
\end{tabular}

For the given problem the heat transfer coefficient $H$ $\left[\mathrm{Wm}^{-2} \mathrm{~K}^{-1}\right]$ describes the quality of the sensor-sample interface with respect to heat transfer over this boundary. In this context the term contact conductance is used. The inverse of the conductance gives the so-called contact resistance. $-\boldsymbol{n}\left(\boldsymbol{q}_{\mathbf{1}}-\boldsymbol{q}_{\mathbf{2}}\right)\left[\mathrm{Wm}^{-2}\right]$ is the net normal heat flux across the boundary in the direction normal to the interface. $T_{\infty}$ correlates to the equilibrium temperature of the sample far away from the source, which is set equal to the initial temperature $T_{\text {init. }}$.

Heat transfer by radiation: especially under low pressure conditions radiation is an important heat transfer mechanism. Thus, radiative interaction between sensor surface and material surface is considered, while radiation inside the medium is not treated. For this purpose the spaces between sensor and sample particles are approximated by a layer with a width of the order of the mean particle radius of the sample material. A schematic sketch is given in Fig. 7. Over the interaction domain bounded by $B 1$ and $B 2$ surface-to-surface radiation heat transfer occurs. For mere transfer between sensor and sample the interaction domain is omitted. At the boundaries $B 1$ and $B 2$ a term $q_{\mathrm{rad}}\left[\mathrm{Wm}^{-2}\right]$ accounts for the net radiation flux at the particular surface.

The boundary condition at the sensor-sample interface $(B 1$ in Fig. 7), given in relation (34), is extended by the radiative term. Therefore, it is composed of two parts, the contact resistance term and the radiative term:

$-\boldsymbol{n}\left(\boldsymbol{q}_{\mathbf{1}}-\boldsymbol{q}_{\mathbf{2}}\right)=H\left(T_{\infty}-T\right)+q_{\mathrm{rad}}$.

At boundary $B 2$ the only modification is due to radiation, thus the boundary condition is

$-\boldsymbol{n}\left(\boldsymbol{q}_{1}-\boldsymbol{q}_{2}\right)=q_{\mathrm{rad}}$.

All models were set up with a sample surrounding the sensor using a continuity boundary condition at the samplecontainer interface and thermal insulation at the outer container wall.

Initial conditions: at $t=0$ the system is at equilibrium (no gradients) and has a preset initial temperature $T_{\text {init }}$. 


\subsection{Model parameters}

Thermal conductivity measurements were simulated for three types of sample conductivity. First, a sample of very low conductivity $\left(0.002 \mathrm{Wm}^{-1} \mathrm{~K}^{-1}\right)$, characteristic for small-grained materials in high vacuum, was considered. Secondly, an intermediate low sample conductivity $\left(0.02 \mathrm{Wm}^{-1} \mathrm{~K}^{-1}\right)$ was investigated and finally a moderate conductivity $\left(0.2 \mathrm{Wm}^{-1} \mathrm{~K}^{-1}\right)$, typical for granular materials under normal pressure of $10^{3} \mathrm{hPa}$, was examined. The other physical parameters describing the sample were not varied. For each of the three sample conductivities, seven different scenarios, as listed in Table 2, were investigated. In these settings the influence of the contact between sensor and sample, axial heat flow along the cables, and radiative energy transport between sensor and sample was examined in detail, as well as a combination of all these factors. The sample and sensor-sample interface parameters used in the numerical simulations are given as follows:

- Sample setting (1): very low sample conductivity: $k=0.002 \mathrm{Wm}^{-1} \mathrm{~K}^{-1}, \varrho=1510 \mathrm{~kg} \mathrm{~m}^{-3}, c=633 \mathrm{~J}$ $\mathrm{kg}^{-1} \mathrm{~K}^{-1}, H=1250 \mathrm{Wm}^{-2} \mathrm{~K}^{-1}$ (good contact), $H=$ $12.5 \mathrm{Wm}^{-2} \mathrm{~K}^{-1}$ (poor contact), $\varepsilon_{\mathrm{sen}}=1.0, \varepsilon_{\mathrm{mat}}=1.0$.

- Sample setting (2): low sample conductivity: $k=0.02 \mathrm{Wm}^{-1} \mathrm{~K}^{-1}, \varrho=1510 \mathrm{~kg} \mathrm{~m}^{-3}, c=633 \mathrm{~J} \mathrm{~kg}^{-1}$ $\mathrm{K}^{-1}, H=1250 \mathrm{Wm}^{-2} \mathrm{~K}^{-1}$ (good contact), $H=12.5$ $\mathrm{Wm}^{-2} \mathrm{~K}^{-1}$ (poor contact), $\varepsilon_{\mathrm{sen}}=1.0, \varepsilon_{\mathrm{mat}}=1.0$.

- Sample setting (3): moderate sample conductivity: $k=0.2 \mathrm{Wm}^{-1} \mathrm{~K}^{-1}, \varrho=1510 \mathrm{~kg} \mathrm{~m}^{-3}, c=633 \mathrm{~J}$ $\mathrm{kg}^{-1} \mathrm{~K}^{-1}, H=1250 \mathrm{Wm}^{-2} \mathrm{~K}^{-1}$ (good contact), $H=$ $12.5 \mathrm{Wm}^{-2} \mathrm{~K}^{-1}$ (poor contact), $\varepsilon_{\mathrm{sen}}=1.0, \varepsilon_{\mathrm{mat}}=1.0$.

The values chosen for material density and specific heat are those of glass beads of $0.25-0.5 \mathrm{~mm}$ grain size (Hütter, 2011, p. 55). Thus, the results of the numerical simulations can be used to analyze the measurement results for those glass beads. The value of contact conductance used for poor contact was taken from Kömle et al. (2008) where it was derived for lunar conditions. As good contact a hundred times better contact conductance was considered. The thermal emissivities of sensor and sample required for radiative interaction were chosen to be those of a black body. In this way the maximum influence of the radiation heat transfer can be assessed.

The applied sample parameters correspond to a thermal diffusivity of $2.1 \times 10^{-9} \mathrm{~m}^{2} \mathrm{~s}^{-1}$ for the very low conductivity, $2.1 \times 10^{-8} \mathrm{~m}^{2} \mathrm{~s}^{-1}$ for the low conductivity and $2.1 \times$ $10^{-7} \mathrm{~m}^{2} \mathrm{~s}^{-1}$ for the moderate conductivity. In Table 3 the transient and maximum measurement times derived for the examined sensors and sample settings are listed.

The heating powers applied for the different sensor types and scenarios were chosen in dependence of the estimated sample thermal conductivity. The criterion was that the temperature rise in response to heating should be no more than
Table 3. Transient and maximum measurement times calculated for the different conductivity and sensor settings.

\begin{tabular}{llll}
\hline & TP02 & LNP01 & LNP03 \\
\hline$R_{\text {sample }}-r_{\text {sen }}$ & $0.1 \mathrm{~m}$ & $0.12 \mathrm{~m}$ & $0.1 \mathrm{~m}$ \\
\hline & $t_{\text {trans }} / t_{\max }$ & $t_{\text {trans }} / t_{\max }$ & $t_{\text {trans }} / t_{\max }$ \\
\hline very low $k$ & $56 \mathrm{~min} / 8.3$ days & $36.7 \mathrm{~h} / 12$ days & $5.1 \mathrm{~h} / 8.3$ days \\
low $k$ & $5.6 \mathrm{~min} / 20$ days & $3.7 \mathrm{~h} / 1.2$ days & $31.5 \mathrm{~h} / 20 \mathrm{~h}$ \\
moderate $k$ & $34 \mathrm{~s} / 2 \mathrm{~h}$ & $22 \mathrm{~min} / 2.9 \mathrm{~h}$ & $3 \mathrm{~min} / 2 \mathrm{~h}$ \\
\hline
\end{tabular}

a few degrees over the entire measurement period. In case of the very low conductivity setting this led to extremely low heating powers of $10^{-3} \mathrm{~W}$ for all sensors. This matches the powers used in the Apollo heat flow experiments (Langseth et al., 1972, 1973).

\subsection{TP02-probe}

The model of the TP02 sensor is made up of six domains, as shown in Fig. 8. Two cylinders, (domain 5 and domain 6) form the needle, one of them representing the heated part, the other representing the unheated part. Attached to the heated part of the needle are the electrical wires modeled as a cylinder made of copper with a diameter of $0.04 \mathrm{~mm}$ and a length of $0.5 \mathrm{~m}$ (domain 1). Diameters of the conducting layers are always chosen in such a way that they approximately match the true wires cross- section, which of course is not necessarily axi-symmetric and therefore cannot be directly modeled in a cylinder-symmetric geometry. The cable is enclosed by the base made of stainless steel (domain 4) and the cable coating made of Teflon (domain 2). Both are modeled as concentric cylinders. Further, the flexible tube protecting the cable-base interface was implemented (domain 3). The measurement point (MP) indicates the location were the temperature response to heating is measured. The physical parameters used for the different sensor domains are given in Table 4 . In the following the results of the numerical simulation for this sensor type are presented.

\subsubsection{Setting (1): very low sample conductivity $\left(k=0.002 \mathrm{Wm}^{-1} \mathrm{~K}^{-1}\right)$}

For the TP02 first, a scenario of a measurement of $36 \mathrm{~h}$ in a material of very low sample conductivity was modeled. Figure 9 shows the derived temperature increase caused by heating for the seven scenarios discussed above. A split up of the heating curves depending on the investigated scenario is observable. The largest temperature increase was found for the setting of pure conduction, no axial heat transport via the cables and poor contact between sensor and sample (No. 1), followed by the temperature curve obtained for the same scenario but with a good contact between sensor and sample (No. 2). 

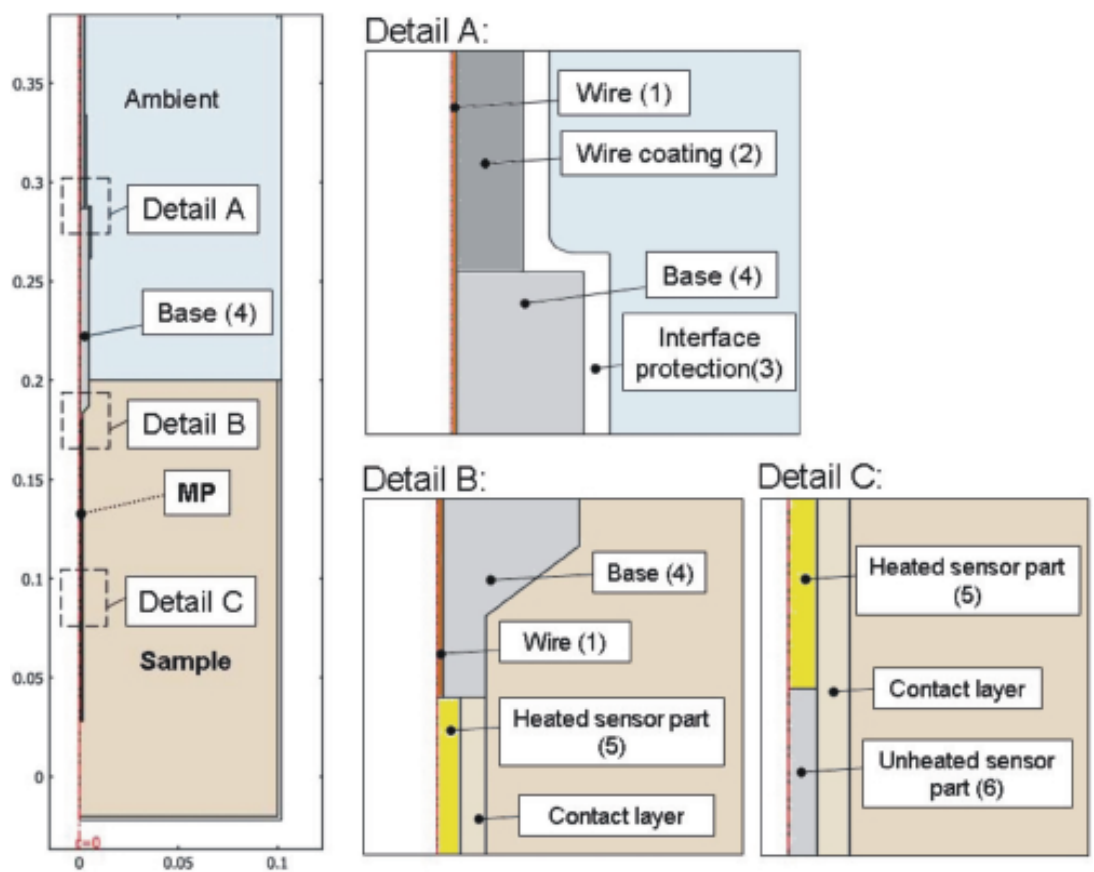

Fig. 8. Model of the TP02 sensor embedded in a sample.

Table 4. Parameters used for the different domains of the TP02-model.

\begin{tabular}{lcccc}
\hline Domain & $\begin{array}{c}T_{\text {initial }} \\
{[\mathrm{K}]}\end{array}$ & $\begin{array}{c}c \\
{\left[\mathrm{~J} \mathrm{~kg}^{-1} \mathrm{~K}^{-1}\right]}\end{array}$ & $\begin{array}{c}\varrho \\
{\left[\mathrm{kg} \mathrm{m}^{-3}\right]}\end{array}$ & $\begin{array}{c}k \\
{\left[\mathrm{Wm}^{-1} \mathrm{~K}^{-1}\right]}\end{array}$ \\
\hline $\begin{array}{l}\text { (1): electrical wires of the sensor } \\
\text { (material: copper) }\end{array}$ & 297 & 385 & 8700 & 400 \\
\hline $\begin{array}{l}\text { (2): insulation of the wires } \\
\text { (material: Teflon (PTFE) }\end{array}$ & 297 & 960 & 2200 & 0.25 \\
\hline $\begin{array}{l}\text { (3): plastic protection of the wires } \\
\text { leaving the base(material: PVC }\end{array}$ & 297 & 960 & 1380 & 0.15 \\
\hline $\begin{array}{l}\text { (4), (5), (6): sensor base, heated } \\
\text { part of the needle and unheated } \\
\text { part of the needle (material: } \\
\text { stainless steel }\end{array}$ & 297 & 500 & 7900 & 16 \\
\hline
\end{tabular}

The weakest temperature response was encountered with the setting including axial heat transport via the cables and radiation heat transport (No. 6 and No. 7), while the temperature increase for the setting of pure conduction and axial losses via the cables (No. 3 and No. 4) is somewhat higher. The case of pure radiative heat transport between sensor and sample (No. 5) without cables yields a temperature curve in between the data obtained for pure conduction with and without axial heat flow allowed.

In the semi-logarithmic representation given in Fig. 9, clear differences in the shape of the temperature curves can be noticed. The data were evaluated using the three methods outlined in Sect. 3.5. For each scenario first, the interval specified by the transient and maximum measurement time was evaluated. In addition, also an early time interval of the measurement curve was evaluated. For the settings No. 1 and No. 2 both evaluation intervals led to values close to the prescribed sample conductivity. For the early time evaluation interval the methods of linear regression and successive linear regression give higher conductivities than for the time period specified by $t_{\text {trans }}$ and $t_{\max }$.

Settings No. 3 and No. 4, where axial heat flow is considered, yield differences in the determined thermal conductivity dependent on the used evaluation method. Best values (closest to sample conductivity) are obtained with the nonlinear fit method, while the other methods deliver higher values. 


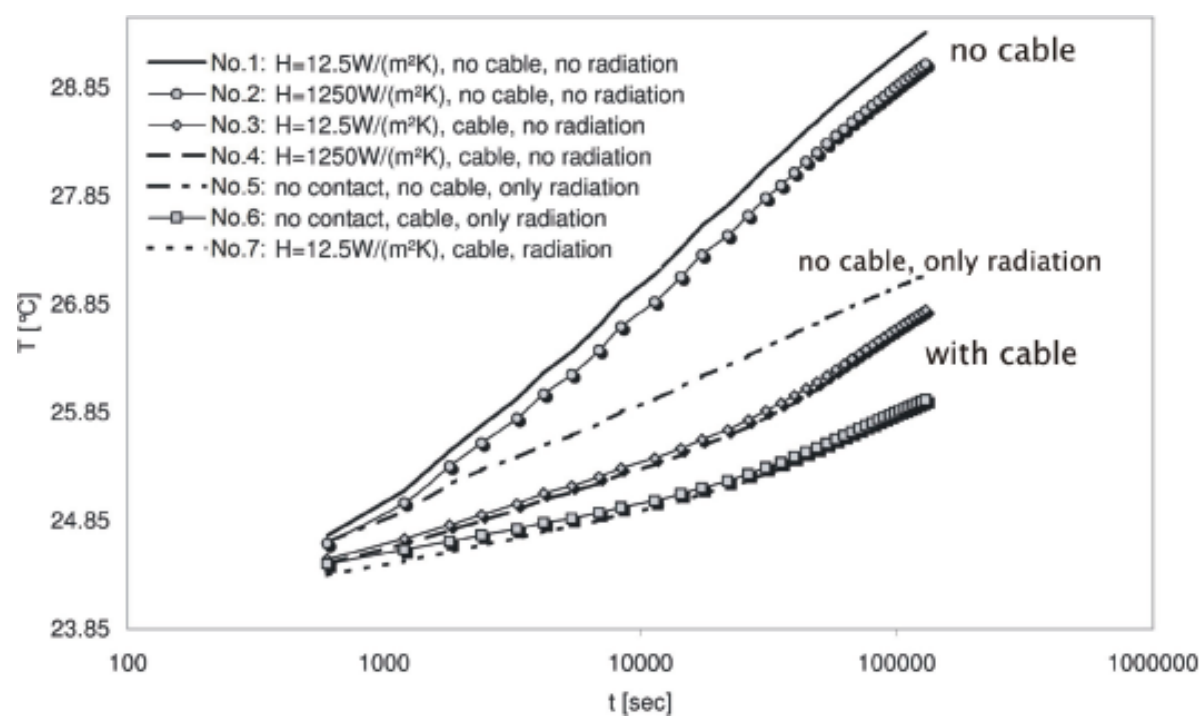

Fig. 9. Temperature response of the commercial TP02-sensor at the measurement point due to heating in a very low conductivity sample with $\lambda=0.002 \mathrm{Wm}^{-1} \mathrm{~K}^{-1}$

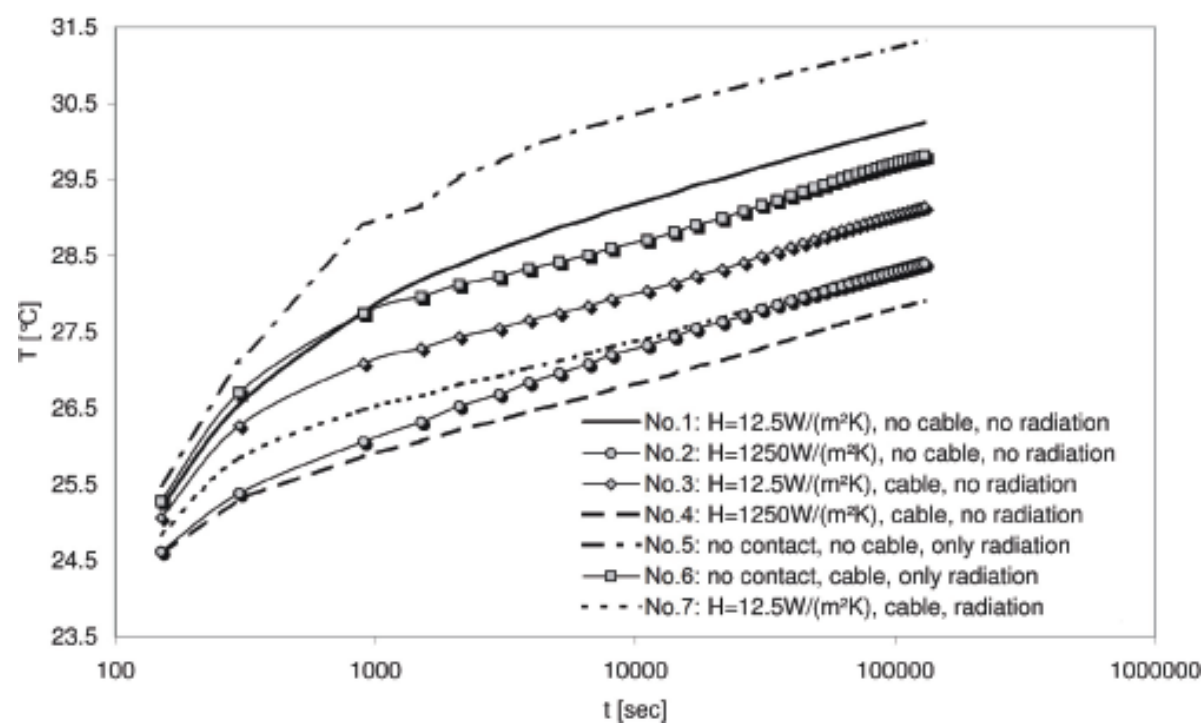

Fig. 10. Temperature response of the commercial TP02-sensor at the measurement point due to heating in a low conductivity sample with $\lambda=0.02 \mathrm{Wm}^{-1} \mathrm{~K}^{-1}$.

Since the measurement curves for these scenarios depict a bend, an additional interval covering the late measurement times was inspected. From this time period, slightly higher thermal conductivities than the sample conductivity were derived by all three methods. The settings involving radiation all yielded significantly higher conductivities than the sample conductivity. From the obtained results several conclusions can be drawn:

- Axial losses via the base and electrical wires result in a smaller temperature increase and a bend of the measurement curve in the semi-logarithmic presentation dividing it in two parts. Separate analysis of these parts gives higher conductivities for the first part and conductivities close to the true sample conductivity for the second part.

- In the sample of very low conductivity, a difference in contact resistance produces a temperature offset, which causes a parallel shift of the measurement curve in the semi-logarithmic presentation. The evaluation of the data yields similar values for the thermal conductivity independent of the contact resistance.

- As recognizable from Fig. 9, the scenario of pure radiation interaction between sensor and sample yields a measurement curve that is shaped different than the 


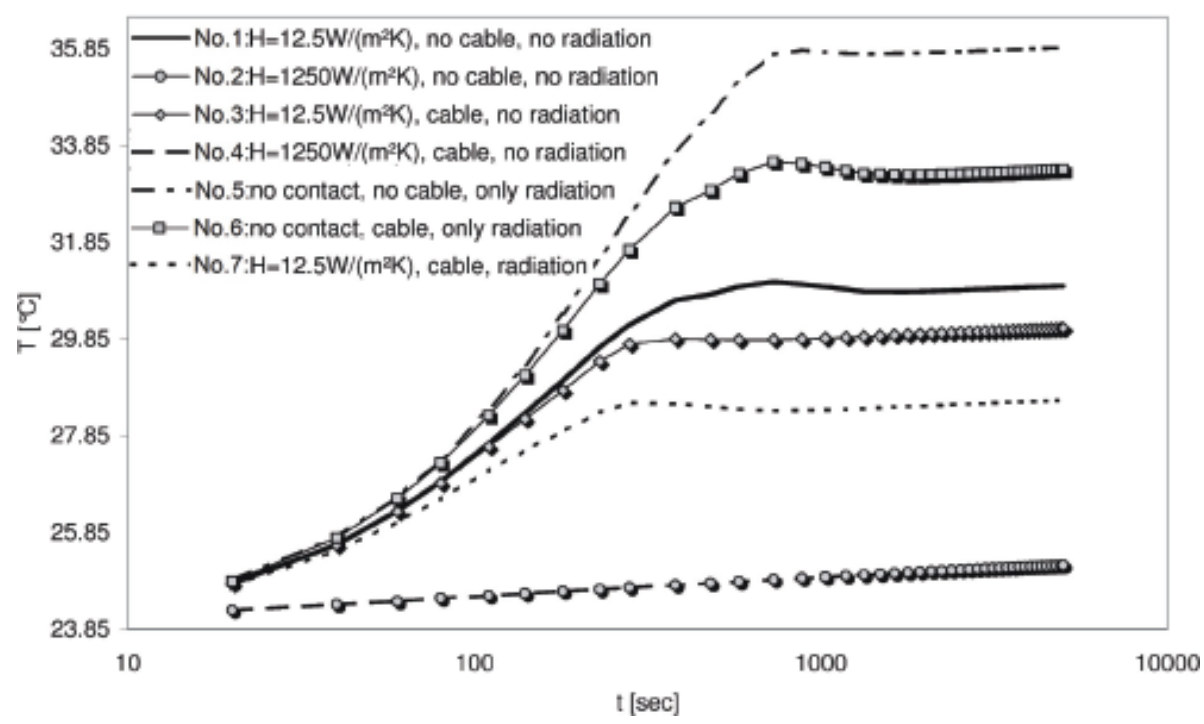

Fig. 11. Temperature response of the commercial TP02-sensor at the measurement point due to heating in a moderate conductivity sample with $\lambda=0.2 \mathrm{Wm}^{-1} \mathrm{~K}^{-1}$.

curves obtained for the other scenarios. The thermal conductivity evaluated from this curve is about twice as high as the preset sample conductivity.

\subsubsection{Setting (2): low sample conductivity $\left(k=0.02 \mathrm{Wm}^{-1} \mathrm{~K}^{-1}\right)$}

For the case of a low conductivity sample measurements of $36 \mathrm{~h}$ were simulated. Figure 10 shows the temperatures derived for the seven different settings. An even split up of the temperature curves depending on the examined scenario can be noted. In the semi-logarithmic presentation the temperature curves appear to be parallel for measurement times larger than about $2.7 \mathrm{~h}(10000 \mathrm{~s})$. The largest temperature increase was found for the setting of pure radiative interaction between sensor and sample and no axial losses occurring (No. 5). The smallest temperature increase was encountered for the scenario of good contact between sensor and specimen and axial heat flow involved (No. 4).

The measurement curves were evaluated in the interval limited by $t_{\text {trans }}$ and $t_{\max }(340$ to $70000 \mathrm{~s})$ and in an early time interval $(600$ to $5000 \mathrm{~s})$. The conductivities derived for the long interval range from 0.023 to $0.032 \mathrm{Wm}^{-1} \mathrm{~K}^{-1}$, which is $15-60 \%$ larger than the prescribed sample conductivity. For the early time interval conductivities from 0.017 to $0.039 \mathrm{Wm}^{-1} \mathrm{~K}^{-1}$ were determined. The smallest conductivities were derived from the temperature response obtained for the setting of pure radiation interaction between sensor and sample without other influences (No. 5). In the early time interval the conductivities determined for the settings considering axial heat flow (No. 3, No. 4, No.6 and No. 7) are larger than those derived from data where axial heat flow was omitted. However, in the conductivities obtained from the in- terval defined by transient and maximum measurement time no such difference can be seen.

- For measurements in low conductivity material axial losses cause a modification of the measured temperature response that leads to higher conductivities.

- In the semi-logarithmic presentation the difference in contact resistance only causes a temperature offset between the measurement curves for measurement times longer than $1000 \mathrm{~s}$.

- For the settings involving radiation, no significant impacts coming from this heat transfer mechanism can be identified.

\subsubsection{Setting (3): moderate sample conductivity $\left(k=0.2 \mathrm{Wm}^{-1} \mathrm{~K}^{-1}\right)$}

For this setting model calculations were done for measurements of $1.5 \mathrm{~h}(5000 \mathrm{~s})$. The determined temperature increase are shown in Fig. 11. The data depict two sorts of curves, which strongly differ in shape and magnitude. One type of measurement data shows a strong partly linear increase in the first half of of the measurement period, followed by an even hump that evolves into a linear part with a comparatively small slope. These data are related to the settings in which radiation or poor contact between sensor and sample or both are considered. The highest temperature increase was found for the scenario of pure radiation heat transport followed by the scenario involving radiative interaction between sensor and sample and axial heat flow via the electrical wires. The second curve type appears linear in the temperature versus $\ln t$ plot over the whole measurement period. These data 


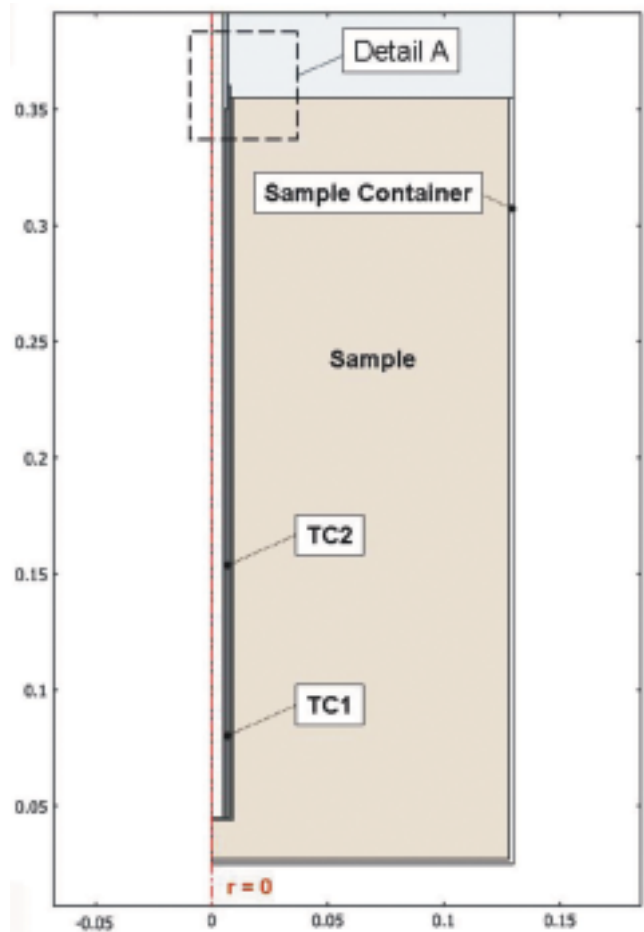

Fig. 12. Model of the LNP01-sensor embedded in a sample.

are related to settings with good contact between sensor and sample. Because of the difference in shape, only the second type measurement curves were evaluated in the interval specified by transient and maximum measurement time. All three evaluation methods deliver conductivities with a maximal deviation of $10 \%$ from the prescribed sample conductivity.

In case of the data of the first shape type, two intervals were evaluated: one covering the strong increase in the first half of the measurement period (100-300 s), the second covering the less steep increase in the second portion of the data $(2000-5000 \mathrm{~s})$. For the first interval conductivities in the range of $10^{-3} \ldots 10^{-2} \mathrm{Wm}^{-1} \mathrm{~K}^{-1}$ were derived. The smallest of those conductivities are related to scenarios considering pure radiation heat transfer, the highest to settings considering radiative heat transport and poor contact. The thermal conductivities determined from the second part of the curves are $10-50 \%$ higher than the sample conductivity. Following points can be noted:

- For measurements in a sample of moderate conductivity poor contact increases the overall magnitude of the temperature increase of a measurement.

- Poor contact increases the transient time.

- For poor contact combined with axial losses via electrical wires, a parallel shift of the temperature increase in the semi-logarithmic representation can be observed.

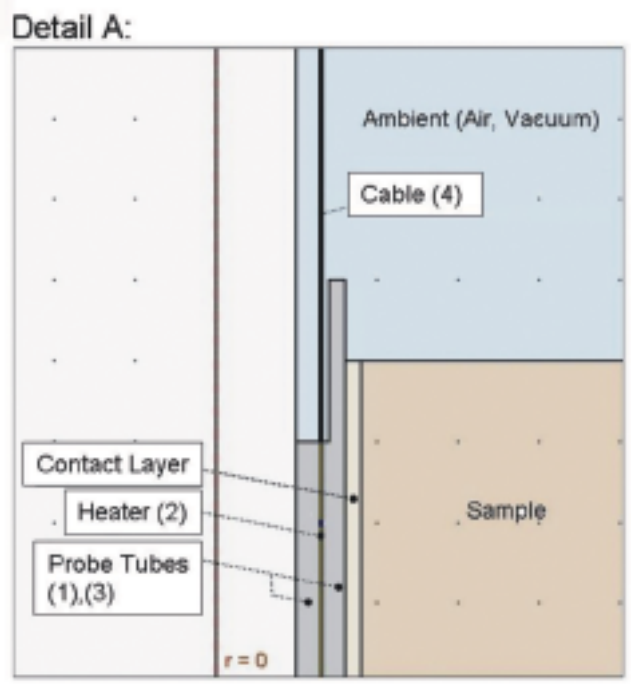

- In case of good contact no differences in temperature time data due to axial heat flow can be observed.

\subsection{LNP01-probe}

The LNP01 sensor was modeled as being composed of three concentric tubes. The middle tube (domain 2) with a thickness of $0.2 \mathrm{~mm}$ and a length of $308 \mathrm{~mm}$ represents the heating foil embedded in the sensor. The adjacent domains $(1,3)$ represent the sensor filling and the sensor walls. The cables of the heater and the thermocouple leaving the sensor were approximated as a concentric cylinder with the same cross section as the true wires and a length of $1 \mathrm{~m}$. The model setup for an LNP01 measurement is shown in Fig. 12. The parameters used for the domains, representing the sensor are listed in Table 5.

\subsubsection{Setting (1): very low sample conductivity $\left(k=0.002 \mathrm{Wm}^{-1} \mathrm{~K}^{-1}\right)$}

Tests with the LNP01 in a very low conductivity material were simulated for measurement periods of $38.8 \mathrm{~h}$ $(140000 \mathrm{~s})$. The temperature responses obtained for the different scenarios are shown in Fig. 13. Similar as in the results obtained for the TP02 very low conductivity simulations, a split up of the measurement curves can be observed. The temperature responses for the different scenarios are accumulated in two groups. The bunch of measurement curves with the higher temperature increase are related 
Table 5. Parameters used for the different domains of the LNP01-model.

\begin{tabular}{lcccc}
\hline Domain & $\begin{array}{c}T_{\text {initial }} \\
{[\mathrm{K}]}\end{array}$ & $\begin{array}{c}c \\
{\left[\mathrm{~J} \mathrm{~kg}^{-1} \mathrm{~K}^{-1}\right]}\end{array}$ & $\begin{array}{c}\varrho \\
{\left[\mathrm{kg} \mathrm{m}^{-3}\right]}\end{array}$ & $\begin{array}{c}k \\
{\left[\mathrm{Wm}^{-1} \mathrm{~K}^{-1}\right]}\end{array}$ \\
\hline $\begin{array}{l}\text { (1),(3): representing inner } \\
\text { and outer steel tube and) } \\
\text { the filling) }\end{array}$ & 297 & 7900 & 500 & 16 \\
\hline $\begin{array}{l}\text { (2): heating foil } \\
\text { (material: Kapton }\end{array}$ & 297 & 1090 & 1420 & 0.12 \\
\hline \begin{tabular}{l} 
(4): wires leaving the sensor \\
\hline
\end{tabular} & 297 & 385 & 8700 & 400 \\
\hline
\end{tabular}

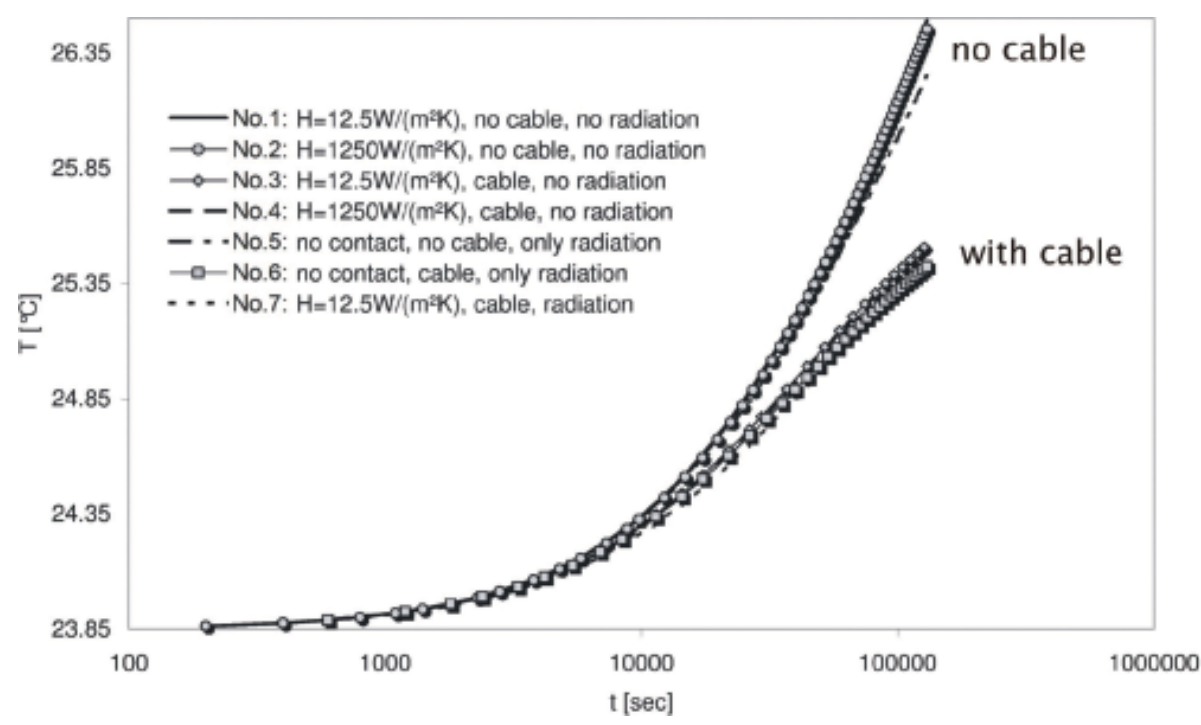

Fig. 13. Temperature response of the hollow cylindrical LNP01-sensor at the measurement point due to heating in a very low conductivity sample with $\lambda=0.002 \mathrm{Wm}^{-1} \mathrm{~K}^{-1}$.

to the measurement settings that do not involve axial heat flow, while the group displaying the smaller temperature rise was calculated for the scenarios including axial heat flow. Moreover, the difference in contact conductance has little effect on the measurement curves, as was found for the TP02 as well. The deviation of the measurement curve determined for the setting of pure radiation heat transport is less pronounced than it is for the TP02. In general, the simulations for the LNP01 indicate that for the very low conductivity setting TP02 and LNP01 show the same behavior. The data were evaluated for three different time periods. First for the time interval specified by $t_{\text {trans }}$ and $t_{\max }(132000-139800 \mathrm{~s})$, secondly for a long interval before the transient time (1500$129900 \mathrm{~s})$ and third an early time period (1500-6000 s).

The time interval prescribed by $t_{\text {trans }}$ and $t_{\max }$ yielded thermal conductivities with a deviation of $10-45 \%$ from the true sample conductivity. The largest deviation was found for settings of pure radiative heat transport with axial losses allowed, followed by the scenarios involving only conduction plus axial heat flow. Except for settings involving radiation, the conductivities derived from the early time interval are sig- nificantly higher than the values calculated from the other time periods. The early time conductivities obtained for settings including radiation interaction (No. 5, No. 6 and No. 7) are similar to the values calculated for the scenarios involving axial heat flow from the time period defined by $t_{\text {trans }}$ and $t_{\max }$.

- For LNP01 measurements in specimen of very low conductivity the development of axial heat flow leads to a smaller overall temperature increase. As for the TP02 the measurement curves depict a different shape of the temperature versus $\ln t$ plot. Axial losses result in higher determined conductivities.

- Differences in contact resistance cause a hardly noticeable shift of the curve.

- In contrast to the results for the TP02, the measurement curves for settings involving radiative heat transport do not appear significantly different in shape and the conductivities derived from these temperature responses are similar to those without radiation. 


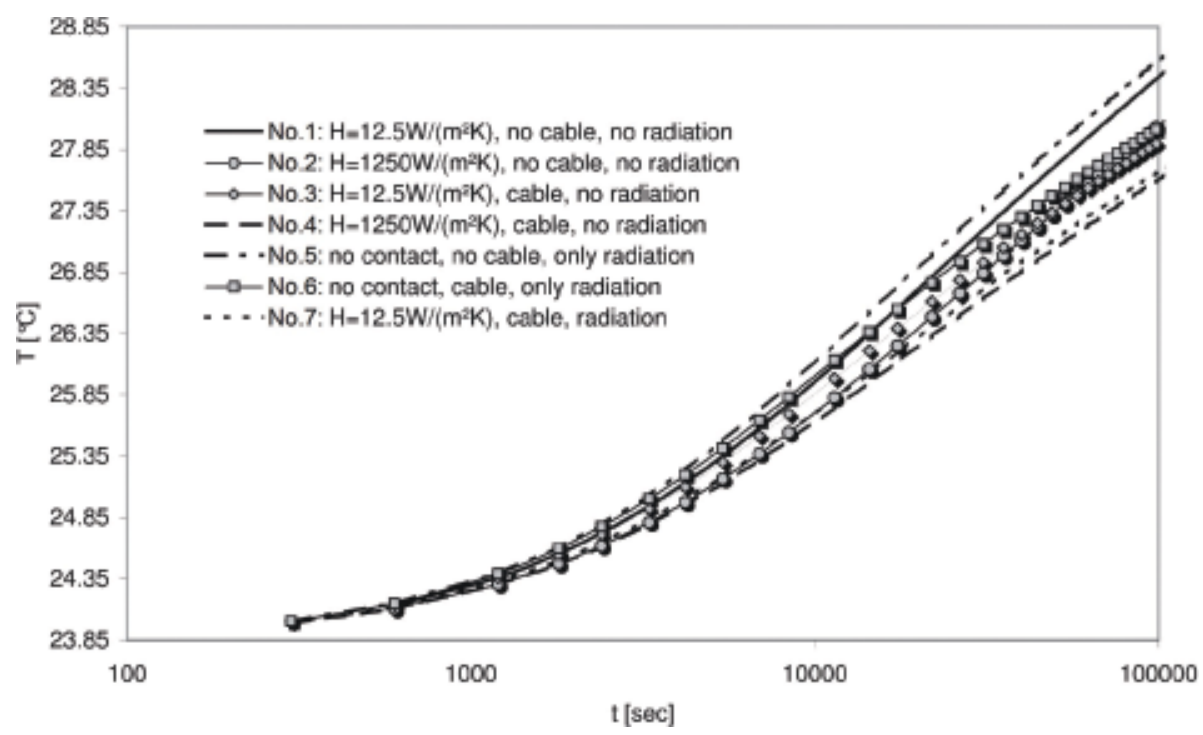

Fig. 14. Temperature response of the hollow cylindrical LNP01-sensor at the measurement point due to heating in a low conductivity sample with $\lambda=0.02 \mathrm{Wm}^{-1} \mathrm{~K}^{-1}$.

\subsubsection{Setting (2): low sample conductivity $\left(k=0.02 \mathrm{Wm}^{-1} \mathrm{~K}^{-1}\right)$}

The setting of a low sample thermal conductivity was investigated for measurement periods of $36 \mathrm{~h}$. The temperature time series derived for the different scenarios are displayed in Fig. 14. As for the TP02 a split up of the measurement curves dependent on the investigated scenarios can be observed. The highest temperature increase was found for the setting of pure radiation heat transport (No. 5), and the lowest for the scenario of good contact between sensor and sample and axial heat flow allowed (No. 4). The data were evaluated in the time interval specified by transient and maximum measurement time and an early time interval. The conductivities determined for the early time interval are considerably higher than those determined from the interval given by $t_{\text {trans }}$ and $t_{\max }$. Further, the conductivities calculated from the early time interval depict a dependence on contact resistance. Good contact leads to higher conductivities than poor contact.

- Axial heat flow influences the measured temperatures in ways that leads to higher evaluated conductivities.

- Differences in contact resistance produce modifications of the temperature response at early measurement times that lead to different conductivities if evaluated. At later times a difference in contact resistance causes constant temperature offset that does not influence the evaluated conductivity.

- The mechanism of radiation heat transport caused no important differences in the measurement curves and evaluated conductivities.

\subsubsection{Setting (3): moderate sample conductivity $\left(k=0.2 \mathrm{Wm}^{-1} \mathrm{~K}^{-1}\right)$}

For the case of a sample material of moderate thermal conductivity, the heat wave introduced by the LNP01 reaches the sample boundaries after $10440 \mathrm{~s}(\approx 2.9 \mathrm{~h})$. Figure 15 shows the temperature increase due to heating for this time period.

A split up into three groups of curves can be observed. The temperature responses with the highest increase are related to the settings considering pure radiation heat transport between sensor and sample. Curves with a moderate temperature increase are associated with the scenarios considering poor contact. The lowest temperature increase was obtained for the settings with good contact between sensor and sample. For all measurement curves the time interval specified by $t_{\text {trans }}$ and $t_{\max }$ was evaluated. For the scenarios with higher temperature increase, which depict a slightly "s-like" shape, in addition an early time period and a late time period were inspected.

- Axial heat transfer via cable connections have only a minor influence on the temperature profile.

- Radiative interaction between sensor and surrounding sample material is negligible compared to the conductive heat transfer.

- A high contact resistance (small conductance) has a strong influence on the temperature profile and tends to underestimate the derived thermal conductivity value.

\subsection{LNP03-probe}

The LNP03 sensor was made up of five domains. Those are a cylinder representing the constantan heating wire (domain 4) 


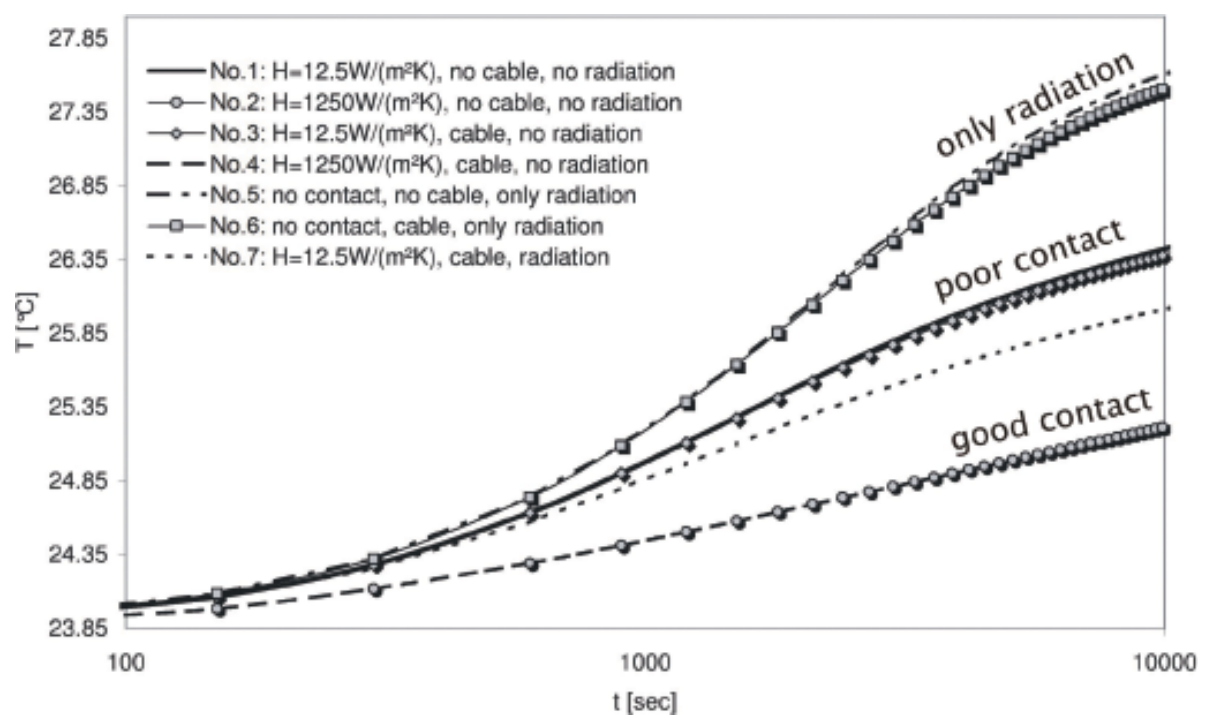

Fig. 15. Temperature response of the hollow cylindrical LNP01-sensor at the measurement point due to heating in a moderate conductivity sample with $\lambda=0.2 \mathrm{Wm}^{-1} \mathrm{~K}^{-1}$.
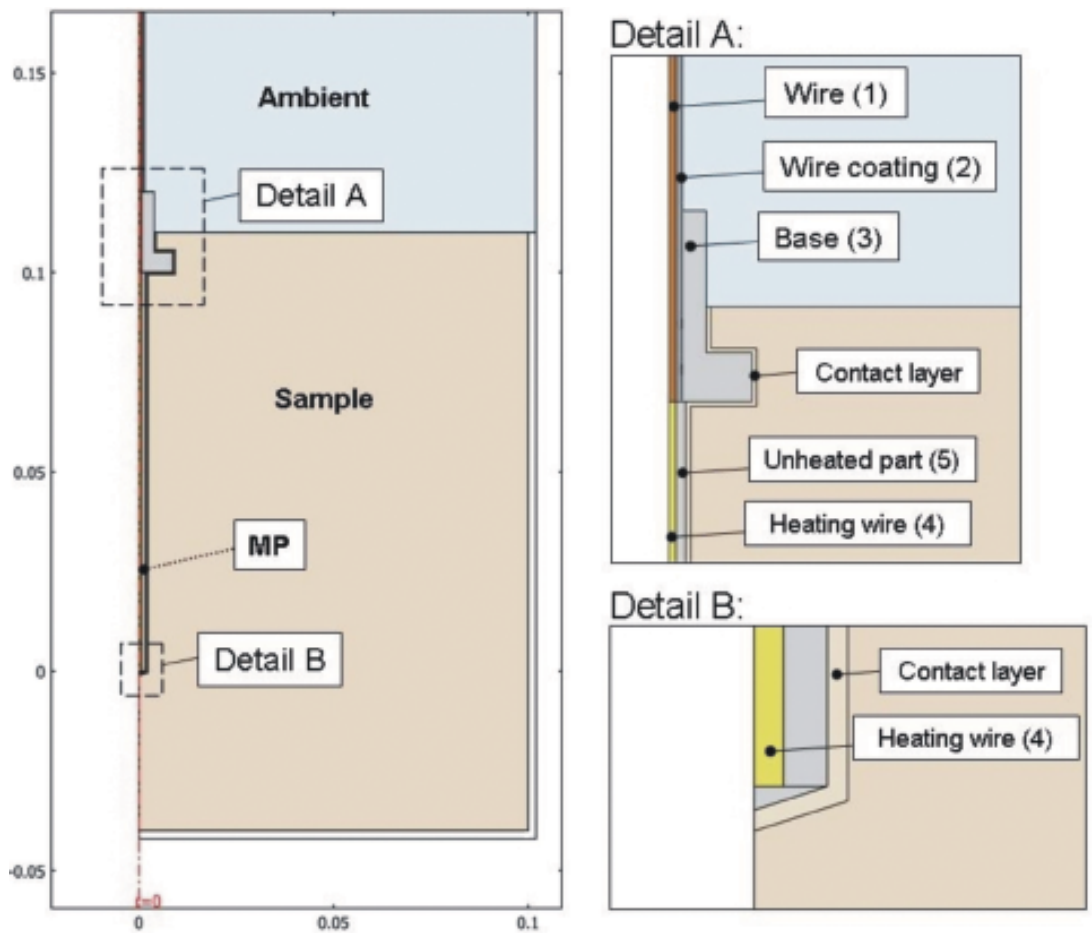

Fig. 16. Model of the LNP03-sensor (short thick needle) embedded in a sample.

in the center, surrounded by a hollow cylinder made of stainless steel (domain 5). Attached to domain (4) for the heating wire are concentric cylinders (domains 1 and 2) that resemble the isolated wires leaving the sensor through the base. The hexagonal part of the base is approximated by a cylinder of the same volume. A schematics of the model is shown in Fig. 16. The parameters used for the domains making up the sensor are listed in Table 6 .

\subsubsection{Setting (1): very low sample conductivity $\left(k=0.002 \mathrm{Wm}^{-1} \mathrm{~K}^{-1}\right)$}

The simulations for LNP03 measurements in a sample of very low conductivity deliver measurement curves similar to those obtained for simulated TP02 measurements in a material of very low conductivity. Measurement periods of $36 \mathrm{~h}$ were simulated. The derived temperature responses are 
Table 6. Parameters used for the different domains of the LNP03-model.

\begin{tabular}{lcccc}
\hline Domain & $\begin{array}{c}T_{\text {initial }} \\
{[\mathrm{K}]}\end{array}$ & $\begin{array}{c}c \\
{\left[\mathrm{~J} \mathrm{~kg}^{-1} \mathrm{~K}^{-1}\right]}\end{array}$ & $\begin{array}{c}\varrho \\
{\left[\mathrm{kg} \mathrm{m}^{-3}\right]}\end{array}$ & $\begin{array}{c}k \\
{\left[\mathrm{Wm}^{-1} \mathrm{~K}^{-1}\right]}\end{array}$ \\
\hline $\begin{array}{l}\text { (1): electrical wires of the sensor } \\
\text { (material: copper) }\end{array}$ & 297 & 385 & 8700 & 400 \\
\hline $\begin{array}{l}\text { (2): plastic protection of the wires } \\
\text { leaving the base (material: PVC) }\end{array}$ & 297 & 900 & 1380 & 0.15 \\
\hline $\begin{array}{l}\text { (3), (5): sensor base and unheated } \\
\text { part of the needle (material: } \\
\text { stainless steel }\end{array}$ & 297 & 500 & 7900 & 16 \\
\hline \begin{tabular}{l} 
(4): constantan heating wire \\
\hline
\end{tabular} & 297 & 410 & 8900 & 49 \\
\hline
\end{tabular}

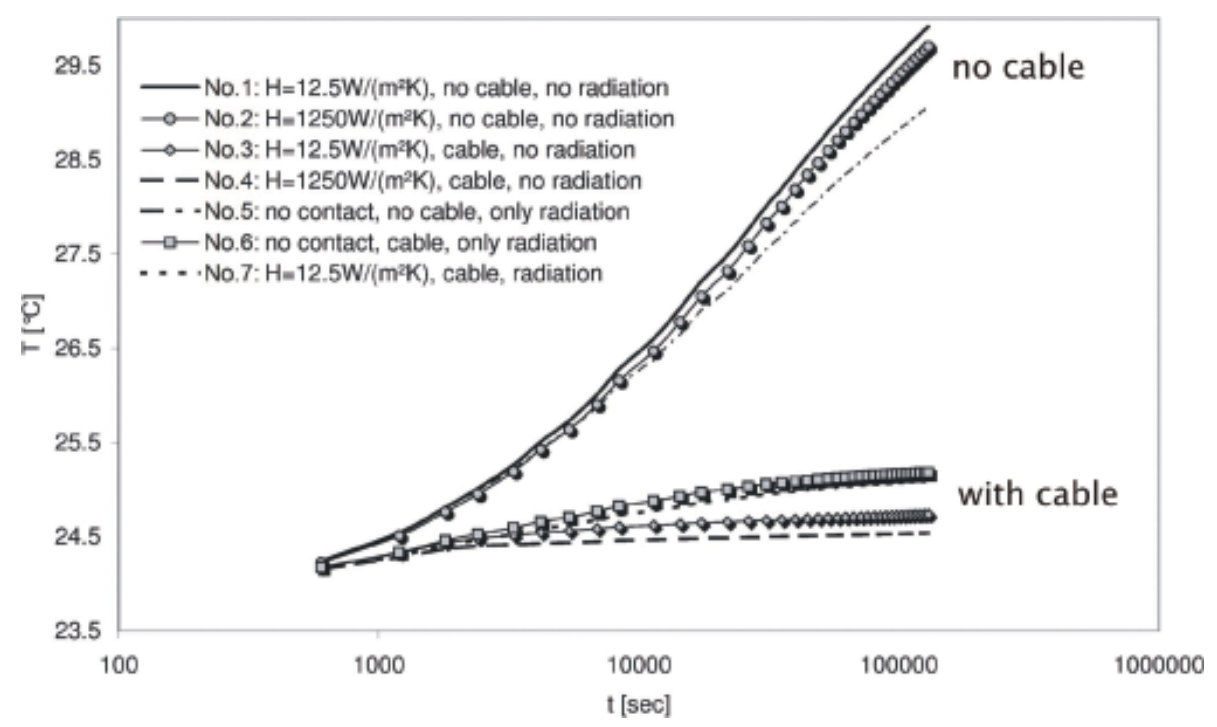

Fig. 17. Temperature response of the LNP03-sensor (short thick needle) at the measurement point due to heating in a very low conductivity sample with $\lambda=0.002 \mathrm{Wm}^{-1} \mathrm{~K}^{-1}$.

shown in Fig. 17. However, no upward bending of the temperature versus time series in the semi-logarithmic plot as for the TP02 was found. Nevertheless, the data are split in three groups. The one with the highest temperature increase corresponds to the settings in which axial heat flow is not included (No. 1, No. 2 and No. 5). The two curves with the lowest temperature increase are related to the scenarios considering pure conduction and axial heat flow (No. 3 and No. 4). The data were evaluated in the time period specified by $t_{\text {trans }}$ and $t_{\max }$. The conductivities obtained from data of settings including axial heat flow are at least ten times larger than the conductivities calculated from the data related to other scenarios. The following conclusions can be drawn:

- Axial heat flow modifies the data such that the evaluation leads to significantly higher conductivities than the true sample conductivity.
- A difference in contact resistance causes a parallel shift of the curves in the semi-logarithmic representation for times larger than $10000 \mathrm{~s}$. This behavior was also found for TP02 and LNP01.

- Radiative heat transfer leads to a somewhat higher temperature increase as the scenarios considering conduction and axial losses only.

\subsubsection{Setting (2): low sample conductivity $\left(k=0.02 \mathrm{Wm}^{-1} \mathrm{~K}^{-1}\right)$}

For the setting of a low sample conductivity measurements of $36 \mathrm{~h}$ were simulated. Figure 18 shows the temperature responses derived for the different settings. Similar as for the TP02 an even split up of the measurement curves can be observed. The highest temperature increase was found for the setting involving only radiation heat transport (No. 5), while the lowest was encountered with the scenario of good contact 


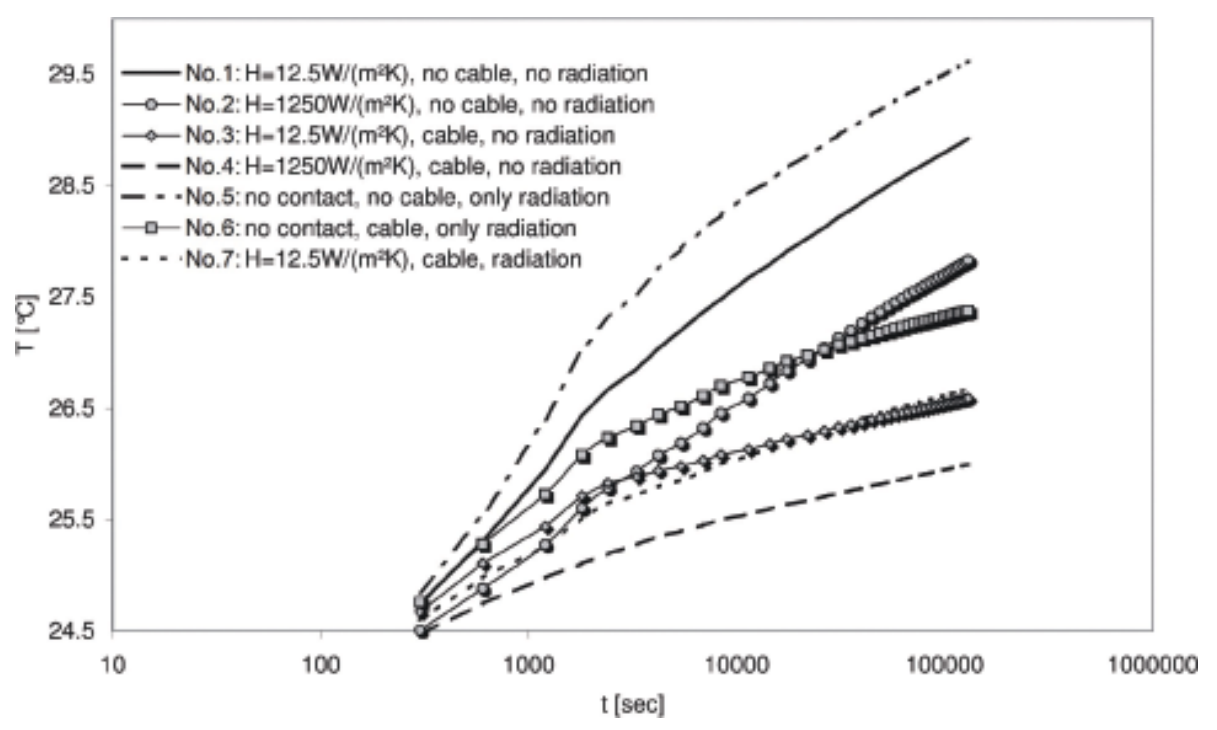

Fig. 18. Temperature response of the LNP03-sensor (short thick needle) at the measurement point due to heating in a low conductivity sample with $\lambda=0.02 \mathrm{Wm}^{-1} \mathrm{~K}^{-1}$.

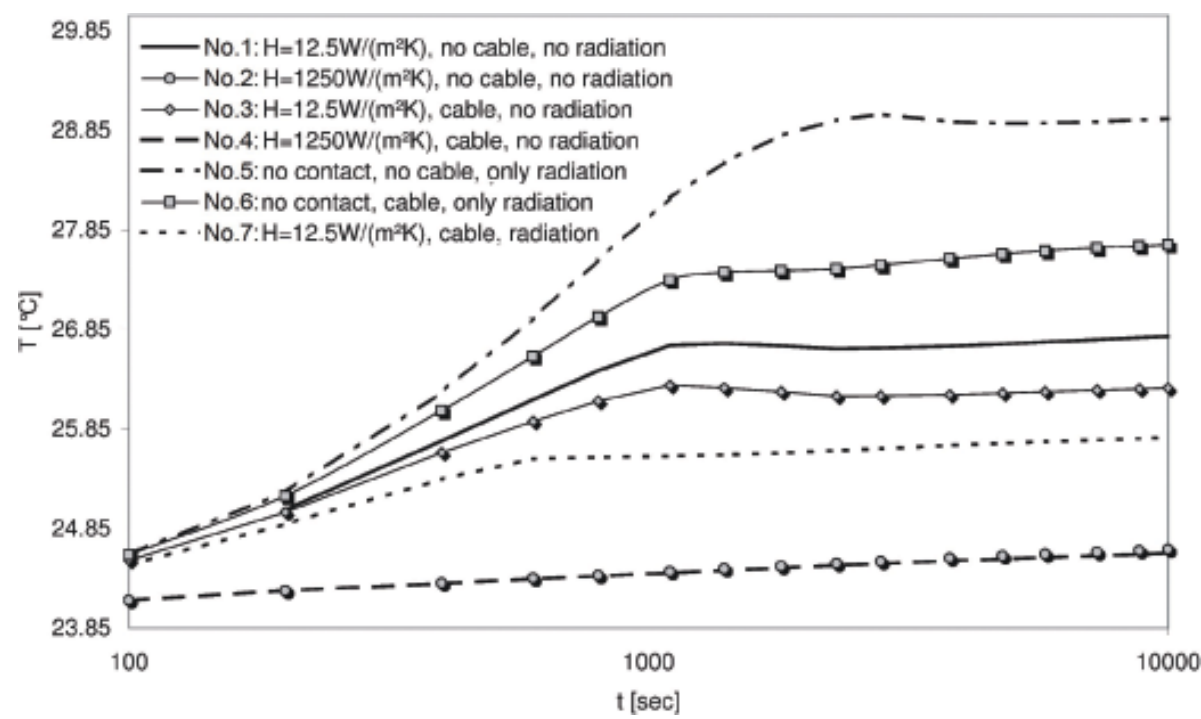

Fig. 19. Temperature response of the LNP03-sensor (short thick needle) at the measurement point due to heating in a moderate conductivity sample with $\lambda=0.2 \mathrm{Wm}^{-1} \mathrm{~K}^{-1}$.

between sensor and sample and axial heat flow occurring. From Fig. 18 it can be seen that the curves obtained from the scenarios including axial heat flow show a different progress than the rest. The data were evaluated in the time interval defined by $t_{\text {trans }}$ and $t_{\max }$. As for the previous setting of a very low sample conductivity, the conductivities calculated from data where axial heat flow was involved (scenarios No. 3, No. 4, No. 6 and No. 7) are significantly higher than the true sample conductivity. The following conclusions arise:

- Axial heat flow leads to lower temperature increases and higher determined conductivities.
- Differences in contact resistance lead to the familiar parallel shift of the curve in the temperature versus $\ln t$ plot.

- Pure radiation interaction between sensor and sample causes no notable differences.

\subsubsection{Setting (3): moderate sample conductivity $\left(k=0.2 \mathrm{Wm}^{-1} \mathrm{~K}^{-1}\right)$}

For LNP03 measurements in a sample of moderate conductivity, measurement periods of $10000 \mathrm{~s}$ were simulated. The received measurement curves are shown in Fig. 19. They exhibit the same behavior as the data obtained for TP02 
measurements in a sample of moderate conductivity. Therefore, the temperature responses obtained for settings No. 2 and No. 4, which are those derived for good contact between sensor and sample, were evaluated using the time period specified by $t_{\text {trans }}$ and $t_{\max }$. In the case of the other curves, two time intervals (an early time period and a late time period) were inspected. The early time interval yielded conductivities about one order of magnitude smaller than the true sample conductivity, while the late time interval gave conductivities in a range similar to the true sample conductivity. From this the following statements arise:

- Axial heat flow leads to enhanced evaluated conductivities.

- A difference in contact resistance causes a change in the initial part of the measurement curve, but at later times it results in a constant temperature offset which does not affect the evaluation.

- Pure radiative interaction between sensor and sample produces the highest temperature increase and somewhat elevated conductivities.

\section{Discussion and conclusions}

Numerical simulations of thermal conductivity measurements with the TP02, LNP01 and LNP03 probe in a sample of varying thermal conductivity, as encountered in a granular material at different pressures, were done. For each conductivity setting different measurement conditions (see Table 2) were studied. A strong dependence of the obtained type of measurement curve on the investigated sample conductivity and measurement condition could be noted. The observed influences of axial heat flow, contact resistance and radiation on the measurements are discussed in the following.

\subsection{Axial heat flow}

The impact of axial heat flow on thermal conductivity measurements depends on the sample thermal conductivity. For very low sample conductivities the largest deviations due to axial losses occur, while for moderate conductivities the influence is negligible. This is caused by the isolating effect of the very low conductivity material. In this case the heat introduced by the measurement flows more easily in axial direction, where the conditions for thermal transport are superior to those prevailing in the sample and thus a large part of the energy is transported along this way. On the other hand, in a sample of moderate conductivity the major portion of the induced heat energy flows via the sample. However, axial losses cause a decrease of the measured temperature response and a modification of the measurement curve that leads to an overestimate of the thermal conductivity, when the standard evaluation methods as presented in Sect. 3.5 are applied. In our simulations the strongest effect due to axial

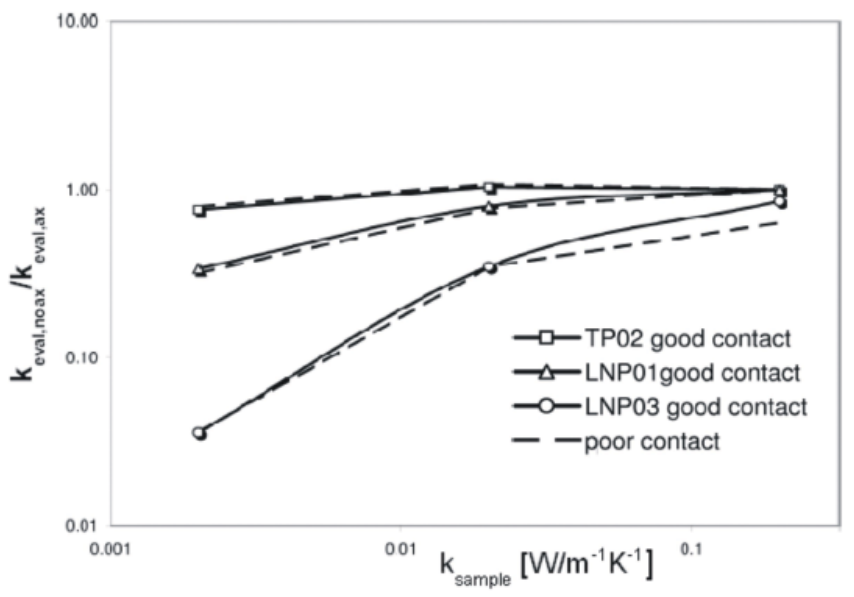

Fig. 20. Ratio of the conductivities determined from the measurement curves, obtained for scenarios including and not including axial heat flow, versus the preset sample conductivity. The continuous lines stand for good contact between sensor and sample and dashed lines for poor contact.

heat flow was encountered with the LNP03, the weakest with the TP02. Figure 20 summarizes the influence of axial heat flow on the determined thermal conductivities as a function of preset sample conductivity.

\subsection{Contact between sensor and sample}

For the simulated conditions a strong influence of the contact conductance (contact resistance) on the measurement curve could be noted for samples of moderate conductivity. A difference in contact conductance causes (i) a difference in the characteristics and length of the transient period and (ii) a constant temperature offset at later measurement times in the semi-logarithmic representation of the measurement curve. Poor contact (low contact conductance) causes a stronger modification of the measurement curve than good contact. Furthermore, the intensity of the influence is controlled by the magnitude of the sample conductivity. If the sample material is a moderate thermal conductor, a high contact resistance (small conductance) acts as a thermal barrier between sensor and sample. In this case the temperature response is modified in the way described above. On the other hand, if the sample itself is a poor conductor, the modification of the measurement curve is negligible. Under such circumstances axial heat flow along the probe axis plays a dominant role and can affect the evaluation results for the thermal conductivity significantly.

\subsection{Radiation heat transport between sensor and sample}

The investigated scenarios, including radiation interaction between sensor and sample, indicate that this heat transfer mechanism can be treated as if there were a high contact 
resistance between sensor and sample. For the settings of low and moderate thermal conductivity, the measurement curves for the radiation scenario showed the strongest modification. Thus, if the radiative interaction between sensor and sample would be replaced by a corresponding contact resistance, it would be even higher than the contact resistance used for poor contact in this work.

\subsection{Evaluation methods}

The nonlinear fit method is the most stable evaluation method and gives the best values also for early time intervals. Influences of too short measurement times and axial heat flow are most severe for the method of linear regression and successive linear regression. Thus the evaluation technique of choice is the nonlinear fit method.

\section{Summary}

In this work sensors suitable for the measurement of the thermal conductivity of soils in situ were investigated for application on surfaces of extraterrestrial bodies like the Moon or Mars. Numerical models of different sensor types were set up, in which measurement scenarios could be studied that were not realizable with the equipment at hand. These simulation data were analyzed in terms of thermal conductivity, using three different evaluation methods, namely linear regression of a predefined interval of the measurement curve, successive linear regression and nonlinear regression.

First, all sensors were tested under laboratory conditions on materials of well known thermal conductivity. By and large all sensors gave consistent results, only the LNP03 and LNP02 probes tend to yield too high thermal conductivities. After that measurements were done on granular materials at air pressure. The obtained data showed an effective thermal conductivity in the order of $10^{-1} \mathrm{Wm}^{-1} \mathrm{~K}^{-1}$ at the normal gas pressure of $10^{3} \mathrm{hPa}$. However, since on many extraterrestrial surfaces reduced pressure conditions are prevailing, thermal conductivity measurements were were also performed on granular materials under varying pressures ranging from air pressure of $10^{3} \mathrm{hPa}$ down to high vacuum of $10^{-4} \mathrm{hPa}$. These results are reported in several separate papers (Hütter et al., 2008; Kömle et al., 2010) and more detailed reports (Hütter et al., 2007; Hütter, 2011). All derived data showed the well known decrease of the effective thermal conductivity with decreasing pressure. However, for pressures below $1 \mathrm{hPa}$, the values obtained from the different sensors for the same material were inconsistent, depicting a dependency on the used sensor.

Therefore, in the present paper this problem was considered in more detail by using a numerical approach simulating the measurements with the particular sensors in materials of varying conductivity. From the results reported it can be seen that for vacuum conditions axial losses play a signif- icant role, while at ambient conditions contact resistance is the main influencing factor. Thus, the results of the numerical simulations suggest that the difference in evaluated thermal conductivity depending on the used sensor at high vacuum is caused by axial losses and too short measurement periods. The model calculations for the different sensors showed that modifications of the measurement curves due to axial losses become most severe for very low conductivities in the range of $10^{-3} \mathrm{Wm}^{-1} \mathrm{~K}^{-1}$, which is the case under high vacuum conditions. Under high vacuum conditions very low heating powers are needed. Furthermore, the necessary measurement times, especially for large sensors in samples of very low conductivity (as e.g. granular materials in vacuum) are up to several days. To fulfill these needs, appropriate data loggers and software, respectively, have to be used.

Moreover, the very low sample conductivities produce severe axial losses in the measurement probes, which lead to significant errors in the evaluated conductivity. This is an important point that needs to be addressed in future work. Further, it follows that thermal conductivity measurements on airless bodies like the Moon using methods based on the transient line heat source technique are strongly influenced by axial losses and need special treatment. For bodies like Mars, where the ambient pressure ranges from 6-10 hPa the simulations reported in this work indicate that such problems would not occur.

Acknowledgements. This work was supported by the Austrian Fonds zur Förderung der wissenschaftlichen Forschung under project L317-N14.

Edited by: A. Benedetto

\section{References}

Abramowitz, M. and Stegun, I. A.: Handbook of Mathematical Functions with Formulas, Graphs, and Mathematical Tables Chapter 5, Dover, 9th Dover Printing, 10th gpo Printing Edn., 1964.

Blackwell, J. H.: A transient flow method for determination of thermal constants of insulating materials in bulk, J. Appl. Phys., 25, 137-144, 1954.

Carslaw, H. S. and Jaeger, J. C.: Conduction of Heat in Solids, Oxford University Press, 2nd Edn., 1959.

de Vries, D. A. and Peck, A.: On the Cylindrical Probe Method of Measuring Thermal Conductivity with Special Reference to Soils, Aust. J. Phys., 11, 255-270, 1958.

Erbas, K.: Eine universelle Methode zur Bestimmung der Wärmeleitfähigkeit aus Aufheizkurven konstant geheizter Zylinderquellen, Ph.D. thesis, Technische Universität Berlin, 2001.

Goodhew, S. and Griffiths, R.: Analysis of Thermal-Probe Measurements Using an Iterative Method to Give Sample Conductivity and Diffusivity Data, Appl. Energ., 77, 205-223, 2004.

Hofmeister, A. M., Pertermann, M., and Branlund, J. M.: Seismology and Structure of the Earth, in: Treatise in 
Geophysics (Vol. 1), edited by: Schubert, G., Elsevier, The Netherlands, 2007.

Hütter, E. S.: Determination of the effective thermal conductivity of granular materials under atmospheric and vacuum conditions, Diploma thesis, 103 pp., Karl-Franzens-University Graz, Austria, 2007.

Hütter, E. S.: Development and testing of thermal sensors for planetary applications, Ph.D. thesis, Karl-Franzens-University Graz, Austria, 129 pp., 2011.

Hütter, E. S., Kömle, N. I., and Kargl, G.: Determination of the effective thermal conductivity of granular materials under varying pressure conditions, J. Geophys. Res., 113, E12004, doi:10.1029/2008JE003085, 2008.

Incropera, F. P., DeWitt, D. P., Bergman, T. L., and Lavine, A. S.: Fundamentals of Heat and Mass Transfer, John Wiley \& Sons, 6th Edn., 2007.

Jaeger, J. C.: Conduction of heat in an infinite region bounded internally by a circular cylinder of perfect conductor, Aust. J. Phys., 9, 167-179, 1956.

Jones, B.: Thermal conductivity probe: development of method and application to a coarse granular medium, J. Phys. E Sci. Instrum., 21, 832-839, 1988.

Kömle, N. I.: Cometary surface processes: Experiments and theory, in: Solar-Planetary Relations, edited by: Biernat, H. K., Lammer, H., Vogl, D. F., and Mühlbachler, S., Research Signpost, Trivandrum, Kerala, India, 2005.

Kömle, N. I., Bing, H., Feng, W. J., Wawrzaszek, R., Hütter, E. S., He, P., Marczewski, W., Dabrowski, B., Schröer, K., and Spohn, T.: Thermal conductivity measurements of road construction materials in frozen and unfrozen state, Acta Geotechnica, 2, 127 138, 2007.

Kömle, N. I., Hütter, E. S., Kargl, G., Ju, H. H., Gao, Y., and Grygorczuk, J.: Development of thermal sensors and drilling systems for application on lunar lander missions, Earth Moon Planets, 103, 119-141, 2008.

Kömle, N. I., Hütter, E. S., and Feng, W. J.: Thermal conductivity measurements of coarse-grained gravel materials using a hollow cylindrical sensor, Acta Geotechnica, 5, 211-223, 2010.

Kömle, N. I., Hütter, E. S., Macher, W., Kaufmann, E., Kargl, G., Knollenberg, J., Grott, M., Spohn, T., Wawrzaszek, R., Banaszkiewicz, M., and Hagermann, A.: In situ methods for measuring thermal properties and heat flux on planetary bodies, Planet. Space Sci., 59, 639-660, 2011.
Koski, J. A. and McVey, D. F.: Application of parameter estimation techniques to thermal conductivity probe data reduction, in: Thermal Conductivity 17 (Proceedings of the 17th International Conference on Thermal Conductivity), edited by: Hust, J., Plenum Press, New York, 587-600, 1983.

Langseth, M. G. J., Clark, S. P. J., Chute, J. L. J., Kheim, S. J. J., and Wechsler, A. E.: Heat flow experiment, in: Apollo 5: Preliminary science report (NASA SP-289), 1972.

Langseth, M. G. J., Kheim, S. J. J., and Chute, J. L. J.: Heat flow experiment, in: Apollo 17: Preliminary science report (NASA SP330), 1973

Mellon, M., Jakosky, B. M., Kieffer, H., and Christensen, P.: HighResolution Thermal Inertia Mapping from Mars Global Surveyor Thermal Emision Spectrometer, Icarus, 148, 437-450, 2000.

Özisik, M. N.: Boundary Value Problems of Heat Conduction, Dover Phoenix Editions, reprinted by David \& Charles, Devon, UK, 1989.

Spohn, T., Seiferlin, K., Hagermann, A., Knollenberg, J., Ball, A., Banaszkiewicz, M., Benkhoff, J., Gadomski, S., Gregorczyk, W., Grygorczuk, J., Hlond, M., Kargl, G., Kührt, E., Kömle, N., Krasowski, J., Marczewski, W., and Zarnecki, J.: Mupus: A thermal and mechanical properties probe for the Rosetta Lander Philae, Space Sci. Rev., 128, 339-362, 2007.

Vos, B.: Analysis of thermal-probe measurements using an iterative method to give sample conductivity and diffusivity data, Appl. Sci. Res., 425-438, 1955.

Wechsler, A.: Compendium of Thermophysical Property Measurement Methods 2, Plenum Press, 1992.

Zent, A. P., Hudson, T. L., Hecht, H., Cobos, D., and Wood, S. E.: Mars regolith thermal and electrical properties: Initial results of the Phoenix thermal and electrical conductivity probe (TECP), in: 40th Lunar and Planetary Science Conference (Lunar and Planetary Science XL), 23-27 March 2009, The Woodlands, Texas, 2009. 